\title{
Repeated genomic signatures of adaptation to urbanisation in a songbird across Europe
}

Pablo Salmón $^{1,2 \dagger}$, Arne Jacobs ${ }^{2 \dagger}$ Dag Ahrén ${ }^{1}$, Clotilde Biard ${ }^{3}$, Niels J. Dingemanse ${ }^{4}$, Davide M. Dominoni $^{2}$, Barbara Helm²,5, Max Lundberg${ }^{1}$, Juan Carlos Senar ${ }^{6}$, Philipp Sprau ${ }^{4}$, Marcel E. Visser $^{5,7}$ and Caroline Isaksson ${ }^{1 *}$

${ }^{1}$ Department of Biology, Lund University.

${ }^{2}$ Current address: Institute of Biodiversity, Animal Health and Comparative Medicine, University of Glasgow, Glasgow, G128QQ, UK

${ }^{3}$ Sorbonne Université, UPEC, Paris 7, CNRS, INRA, IRD, Institut d'Écologie et des Sciences de l'Environnement de Paris, iEES Paris, F-75005 Paris, France.

${ }^{4}$ Department of Biology, Ludwig Maximilians University Munich, Germany.

${ }^{5}$ GELIFES - Groningen Institute for Evolutionary Life Sciences, University of Groningen, The Netherlands.

${ }^{6}$ Museu de Ciències Naturals de Barcelona, Barcelona, Spain.

${ }^{7}$ Department of Animal Ecology, Netherlands Institute of Ecology (NIOO-KNAW), Wageningen, Netherlands.

*Correspondence to Caroline Isaksson, Caroline.Isaksson@biol.lu.se

$\dagger$ Both authors contributed equally 


\section{$\underline{\text { Abstract }}$}

Urbanisation is currently increasing worldwide, and there is now ample evidence of phenotypic changes in wild organisms in response to this novel environment, but the extent to which this adaptation is due to genetic changes is poorly understood. Current evidence for evolution is based on localised studies, and thus lacking replicability. Here, we genotyped great tits (Parus major) from nine cities across Europe, each paired with a rural site, and provide evidence of repeated polygenic responses to urban habitats. In addition, we show that selective sweeps occurred in response to urbanisation within the same genes across multiple cities. These genetic responses were mostly associated with genes related to neural function and development, demonstrating that genetic adaptation to urbanisation occurred around the same pathways in wildlife populations across a large geographical scale. 


\section{Main}

Urban development is rapidly expanding across the globe, and although urbanisation is regarded a major threat for wildlife (Hendry, Gotanda, and Svensson 2017), its potential role as an evolutionary driver of adaptation has not been explored until recently (Johnson and MunshiSouth 2017; J. C. Mueller et al. 2013; Harris and Munshi-South 2017; Rivkin et al. 2019). Some species have phenotypically adapted to the many urban challenges, such as higher levels of noise, artificial light at night, air pollution, altered food sources and habitat fragmentation (Alberti et al. 2017). Indeed, there is now evidence that some of these adaptations may have a genetic basis (Campbell-Staton et al. 2020), in line with the finding that such micro-evolutionary adaptations can occur within short timescales, particularly in response to human activities (Bosse et al. 2017; Hendry, Farrugia, and Kinnison 2008). However, the short evolutionary timescale, the dependence of evolution on local factors, and the polygenic nature of many phenotypic traits, make detecting evolutionary signals difficult (Hendry, Farrugia, and Kinnison 2008; Pritchard, Pickrell, and Coop 2010). Thus, we still lack important knowledge on the signals of adaptation, and thereby of the actual magnitude of the evolutionary change induced by urbanisation on wildlife populations.

The majority of available studies on the genetic bases of urban adaptation have either focused on a limited number of markers and genes (J. C. Mueller et al. 2013) or focused on a narrow geographical scale (Harris and Munshi-South 2017; Campbell-Staton et al. 2020; Perrier et al. 2018). As a result, an important gap remains in the understanding of the prevalence of convergent evolution among cities (Rivkin et al. 2019), limiting the inferences that can be made on the genomic response to urbanisation. A robust approach to address this gap is the use of paired urban and rural sites at a large spatial scale in combination with high throughput genomic tools. Such a strong replicated approach can help to simultaneously detect subtle allele frequency shifts and identify genomic regions repeatedly involved in parallel evolutionary adaptation or under divergent selection across distant urban habitats. This approach provides a powerful framework to test the repeatability of the genomic adaptation to urbanisation.

In this study, we present a multiple location analysis of the evolutionary response to urbanisation, using the great tit (Parus major). This widely-distributed passerine bird is a model species in urban, evolutionary and ecological research (e.g. Boyce and Perrins 1987; 
Charmantier et al. 2008; 2017; Pettifor, Perrins, and McCleery 1988; Bouwhuis et al. 2009; Krebs 1971; Salmón et al. 2017; Sprau, Mouchet, and Dingemanse 2017; Senar et al. 2017; Isaksson et al. 2009), with demonstrated phenotypic changes in response to urban environments in several populations (Charmantier et al. 2017; Sprau, Mouchet, and Dingemanse 2017; Senar et al. 2017; Caizergues, Grégoire, and Charmantier 2018). Additionally, genomic resources are well developed for this species (Kim et al. 2018) and it is known that across its European range, the species presents low genetic differentiation (Perrier et al. 2018; Laine et al. 2016; Lemoine et al. 2016; Spurgin et al. 2019). In order to examine and test the repeatability in the genomic responses to urbanisation in a broad geographical scale we analysed nine paired urban and rural great tit populations across Europe (Figs. 1a and 1b; Table S1). All urban sites used in the study were located in built-up areas or parks within the city boundaries, while rural sites were always natural or semi-natural forests containing only a few isolated houses. Additionally, we quantified the relative degree of urbanisation for each site (urbanisation score: $\mathrm{PC}_{\mathrm{urb}}$, from principal component analysis, PCA; see Methods and Materials, Table S1; Fig. 1b). We combine two complementary approaches, the detection in urban habitats of parallel allele frequency shifts across many loci and the search for independent and repeated selective sweeps (i.e. a strong increase in haplotype frequency at one or few loci). Furthermore, we use functional enrichment analyses of genes putatively under selection to infer which particular phenotypes, known and unknown, are associated with genes under selection in urban great tits, providing an outline of genes to target in future ecological and functional studies.

\section{$\underline{\text { Results and Discussion }}$}

Genetic diversity and population structure across European urban and rural populations

A total of 192 individuals were genotyped at 526,528 filtered SNPs, with 10-16 individuals per site (Table S1). The genetic diversity between each urban-rural pairing was relatively low, with genome-wide differentiation $\left(\mathrm{F}_{\mathrm{ST}}\right)$ ranging between 0.004 to 0.050 (Fig. S1c; Table S1). This is in line with previous studies of the species (Perrier et al. 2018; Laine et al. 2016; Lemoine et al. 2016; Spurgin et al. 2019). In addition, the levels of heterozygosity were similar between urban and rural populations, although slightly lower in some of the urban populations (see Table $\mathrm{S} 1$ for details; Wilcoxon test: $\mathrm{W}=30, \mathrm{P}=0.377$ ). This indicates that the colonisation of urban environments is likely not a result of strong bottlenecks and its associated 
loss of genetic diversity. Despite the low population structure (Laine et al. 2016), two populations at the edge of the species distribution range (Lisbon and Glasgow) separated from all other populations along $\mathrm{PC} 1_{\mathrm{Gen}}$ and $\mathrm{PC} 2{ }_{\mathrm{Gen}}$ (Fig. 1c; Figs. S1a and S2b). This pattern is possibly a consequence of the slightly reduced heterozygosity in these two populations (Figs. 1d and S3; Table S1). However, overall the population structure analyses suggest that urban-rural population pairs from the different localities have colonised each urban habitat independently, as urban-rural pairs split separately along $\mathrm{PC}_{\mathrm{Gen}}$ (Fig. S2a), though still certain number of the urban populations cluster together.
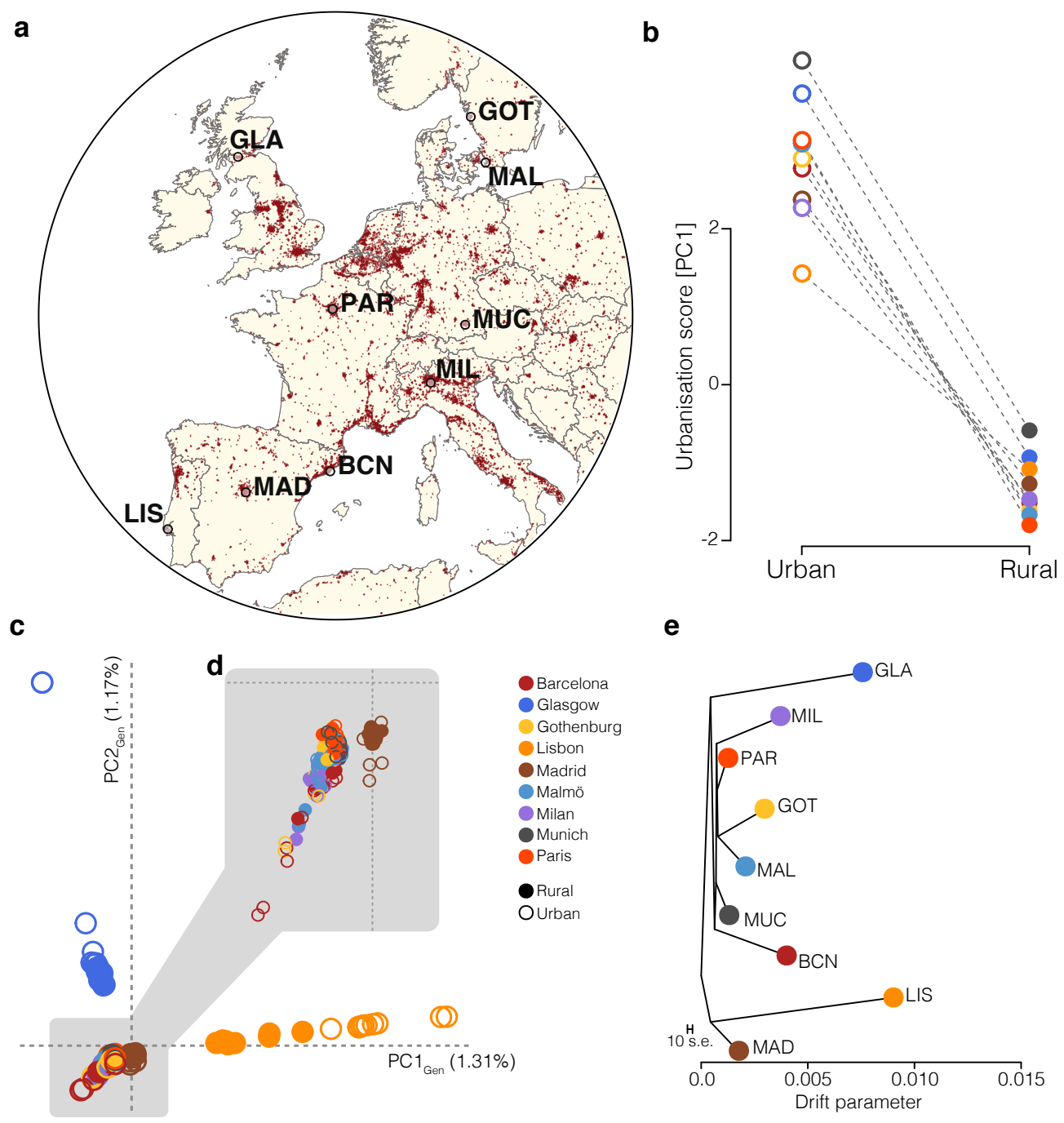

C e

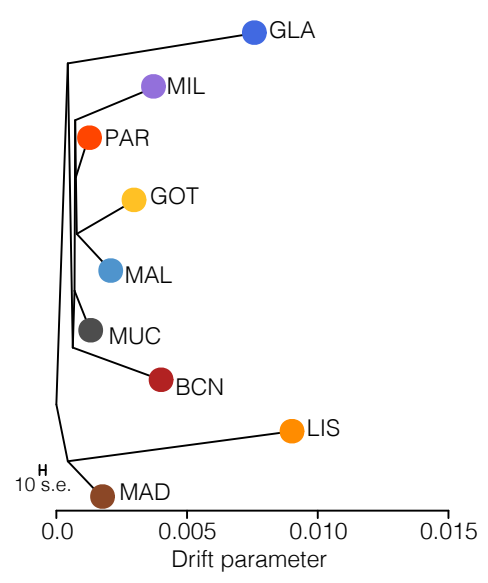

Fig. 1. Urbanisation and population structure. a, Map of Europe, showing the targeted cities where the sampling of great tits (Parus major) was carried out. Red area indicates main dense urban areas. Shapefiles obtained from Natural Earth (https://www.naturalearthdata.com ). b, Urbanisation scores 
(principal component, $\mathrm{PC}_{\text {urb }}$ ) for all nine urban-rural pairings. c, d, Principal component $\left(\mathrm{PC}_{\mathrm{Gen}}\right)$ plot showing the main axes of population structure for European great tits; dots represent individual birds. d, Zoomed view into the population cluster highlighted by the grey-shaded area. e, Maximum-likelihood tree showing the relationship between tits across sampling localities. BCN: Barcelona; GLA: Glasgow; GOT: Gothenburg; LIS: Lisbon; MAD: Madrid; MAL: Malmö; MIL: Milan; MUC: Munich; PAR: Paris.

\section{Identification of key SNPs associated with urbanisation}

Despite the overall lack of genome-wide differentiation between urban-rural pairings, the selective pressures associated with urbanisation might have led to detectable genomic signatures of local adaptation. We used two complementary approaches, LFMM (Latent Factor Mixed Models) and an additional Bayesian approach (using BayPass), to narrow down genomic regions with consistent and strong allele frequency shifts associated with urbanisation. Testing for genotype-environment associations with $L F M M$ (using $\mathrm{PC}_{\mathrm{urb}}$ as a continuous habitat descriptor, Fig. $1 b)$ revealed 2,758 SNPs associated with urbanisation $(0.52 \%$ of the full SNP dataset, falsediscovery rate $(\mathrm{FDR})<1 \%$; Fig. 2a). These SNPs were widely distributed across the genome and did not cluster in specific regions. Larger chromosomes contained more urbanisation-associated SNPs $\left(\mathrm{R}^{2}=0.97\right.$; Fig. $\left.2 \mathrm{c}\right)$, highlighting the polygenic nature of urban adaptation. A PCA based on these SNPs clearly separated urban and rural populations along PC1 $1_{\text {LFMM }}$ (Proportion of variance explained (PVE) by PC1 = 1.98\%; Fig. S4a), showing highly parallel allele frequency changes in those loci across European cities. BayPass identified 70 urbanisation-associated SNPs (Bayes factor $\geq 20$; Fig. 2b), of which 34 were shared with the LFMM analysis. These shared SNPs, which we term "core urbanisation SNPs" (Fig. 2d; Table S2), are likely involved in the local adaptation of great tits to urban habitats, and indeed, they strongly discriminated urban and rural individuals across Europe (PVE by $\mathrm{PC} 1_{\mathrm{GWAS}}=11.7 \%$; Figs. $2 \mathrm{e}$ and $\mathrm{S} 4 \mathrm{~b}$ ).

The importance of habitat (i.e. urban versus rural) was further underpinned by a univariate linear model. Using the first principal component axis of urbanisation-associated SNPs (PC1 $\left.1_{\mathrm{GWAS}}\right)$, habitat explained $73 \%$ of the total associated variance in allele-frequency divergence $\left(\eta^{2} \mathrm{PC1}\right.$ GWAS, Habitat $\left.=0.73, \mathrm{P}<0.001\right)$. In comparison, both the effect of locality, which corresponds to the distinct evolutionary history of local populations ( $\eta^{2}$ PC1 GWAS, Locality $=0.20, P$ $<0.001$ ), and the interaction of locality and habitat, which describes differences in the direction of allele-frequency change across cities $\left(\eta^{2} \mathrm{PC} 1\right.$ GWAS, Habitat $\times$ Locality $\left.=0.13, \mathrm{P}=0.001\right)$, explained much lower proportions of the variance than the habitat term on itself. Interestingly, the 
trajectories of minor allele frequency changes for the individual "core urbanisation SNPs" were highly repeatable across populations (Fig. S5). Thus, the directionality and/or magnitude in allele frequency of the 34 identified SNPs showed a highly parallel pattern across all urban populations (Fig. 2f), suggesting that in this species, local adaptation to urban habitats has occurred through repeated shifts in allele frequency of the same loci. This finding implies an important role of standing genetic variation in urban adaptation of great tits, as putatively adaptive alleles were shared across large parts of Europe (Spurgin et al. 2019).

a

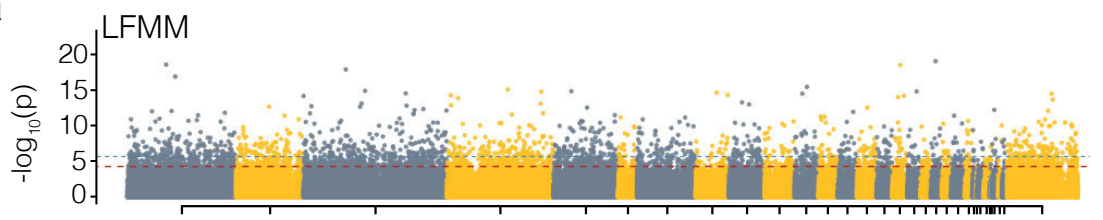

b
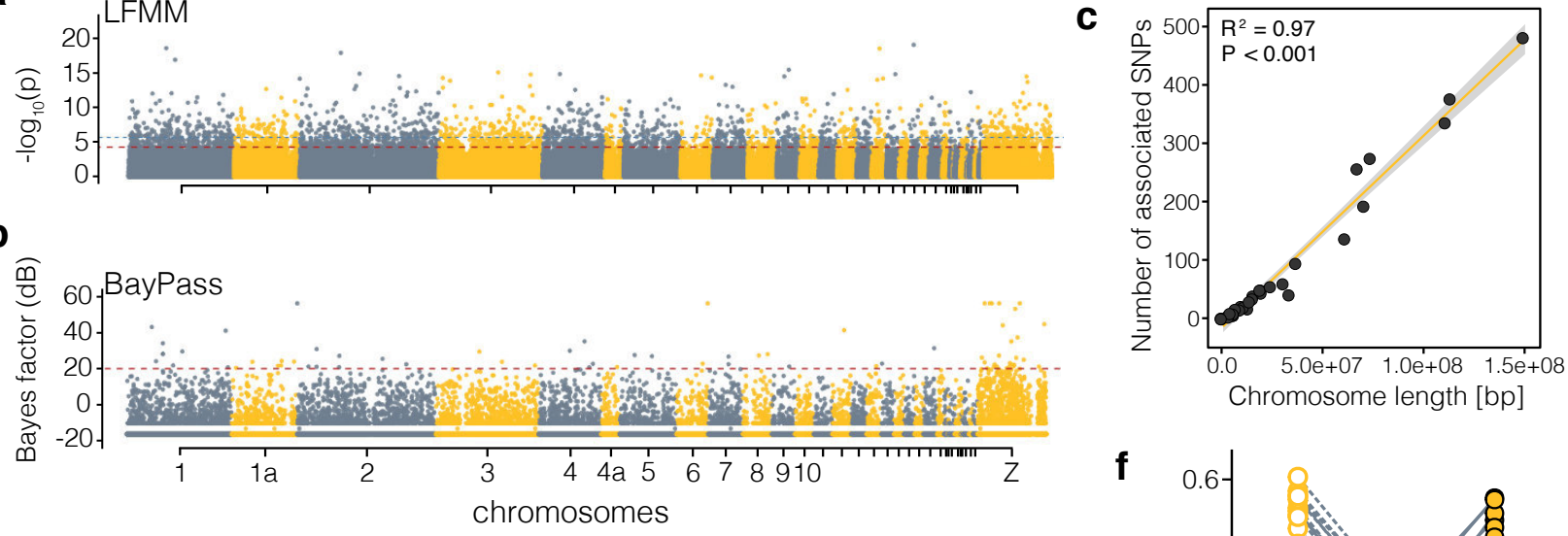

d

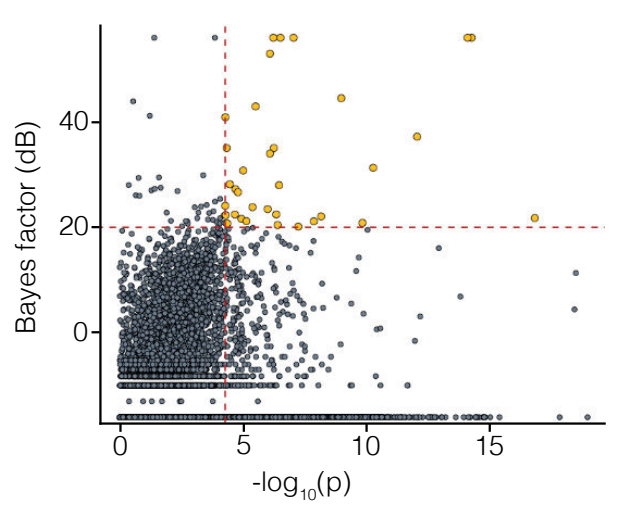

e
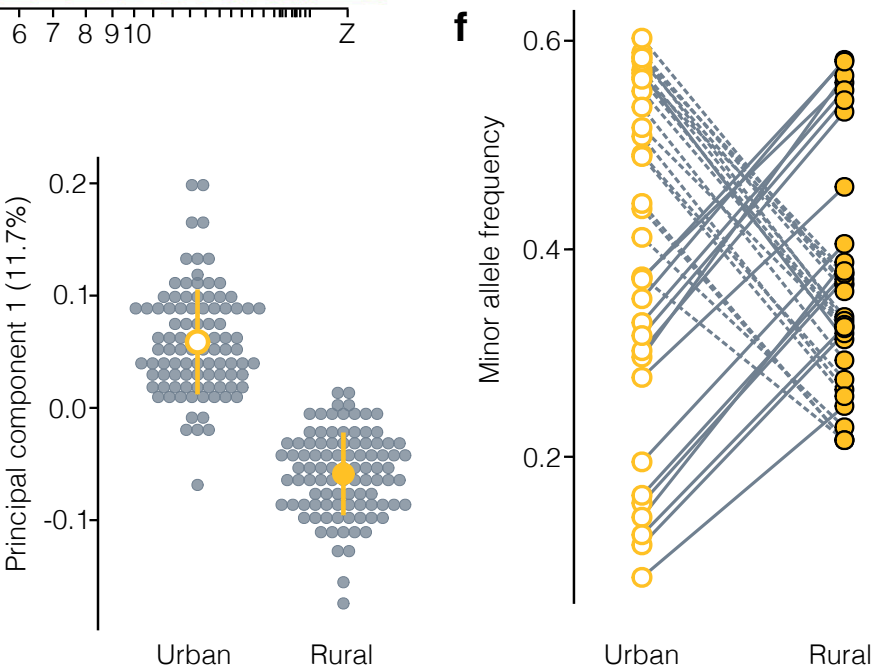

Fig. 2. Genome-wide association with urbanisation. $\mathbf{a}$ and $\mathbf{b}$, Manhattan plots showing signals of genome-wide association with urbanisation across all populations for the $\mathbf{a}, L F M M$ and $\mathbf{b}$, BayPass analyses, respectively (see in methods "Environment-associated SNPs"). The red dotted line and the blue line in the LFMM Manhattan-plot show the 0.1 and 1\% FDR significance thresholds. The red dotted line in the BayPass plot shows the significance threshold for a Bayes factor of 20 deciban (dB); alternating colors denote chromosomes. c, Correlation between the number of SNPs associated with urbanisation per chromosome in the $L F M M$ analyses and the respective chromosome length. The strong correlation indicates a polygenic basis of urban adaptation. d, Correlation between association signals in $L F M M$ and BayPass. The red dotted lines show the respective significance thresholds (BayPass: 20db; LFMM: 1\% 
FDR). SNPs associated with urbanisation in both analyses are highlighted in yellow ("core urbanisation SNPs", N=34). e, Main axis of variation in a PCA ${ }_{\text {GWAS }}$ based on "core urbanisation SNPS". Grey circles show individuals and yellow dots and lines, show the mean \pm s.d. for urban and rural populations. f, Reaction norm plot showing the difference in the mean minor allele frequency for "core urbanisation SNPS" between all urban and rural populations combined. See Fig. S5 for allele frequency trajectories by locality and SNP.

\section{Signatures of selection in urban populations}

Next, we performed a genome-wide scan of differentiation and selective sweep analyses to identify putative signatures of divergent selection between urban and rural populations. We first estimated genetic differentiation $\left(\mathrm{F}_{\mathrm{ST}}\right)$ in $200 \mathrm{~kb}$ sliding windows and $50 \mathrm{~kb}$ steps across the genome. While the genome-wide level of differentiation was low in all populations, we detected highly variable landscapes of genetic differentiation between adjacent urban and rural populations, with multiple genomic peaks of increased genetic differentiation $\left(\mathrm{F}_{\mathrm{ST}}>99^{\text {th }}\right.$ percentile) spread across the genome (Fig. S6). 85 genomic windows were significantly differentiated in at least three urban-rural pairs.

Genetic differentiation can be driven by a myriad of processes, including background selection (selection against deleterious variants) or divergent selection (e.g. associated with local adaptation) in urban and/or rural populations (Burri 2017). To narrow down these processes, we determined whether the outlier windows were putatively under selection in urban populations by estimating population branch statistics $(P B S)$ for each urban population in the same genomewide windows. In this study, positive $P B S$ values show an extended genetic distance of the urban population compared to the adjacent rural population and outgroup in a specific genomic window, indicative of positive selection in the urban population at that site (Yi et al. 2010). We used the rural population from Lisbon as the outgroup for estimating $P B S$, except for Lisbon, for which we used the rural population from Glasgow. We tested the effect of outgroup on $P B S$ values but found that these were generally significantly correlated for each city (average spearman's $r=0.525 \pm 0.087$ s.d., Fig S7 and S8). Between $25 \%$ and $50 \%$ of differentiated genomic regions ( $\mathrm{F}_{\mathrm{ST}}$ ) showed signs of selection based on $P B S$ (top 1\% of empirical $P B S$ distribution) in urban populations (Fig. 3). Taking into consideration the nature of the urbanisation phenomenon, we might expect that the distinct genomic backgrounds would give rise to independent selective sweeps across localities, i.e. the result of locality-specific selection pressures. Yet, the shared genetic variation in this species across Europe(Spurgin et al. 2019) 
might lead to the re-use of shared adaptive variants across geographically distant populations. $P B S$ values were highly variable across locations and showed generally low correlations among each other $(|\mathrm{r}|<0.28)$ suggesting that putative selective sweeps are geographically limited, i.e. locality-specific. Nonetheless, 75 genomic windows with elevated $P B S$ values were shared across more than three urban populations (maximum of five localities, Fig 3). At the gene-level, we detected multiple genes in or close to windows putatively under selection (within $\leq 100 \mathrm{~kb}$ ) across urban populations, of which five genes showed signs of selection in five out of nine urban populations (Table S3).

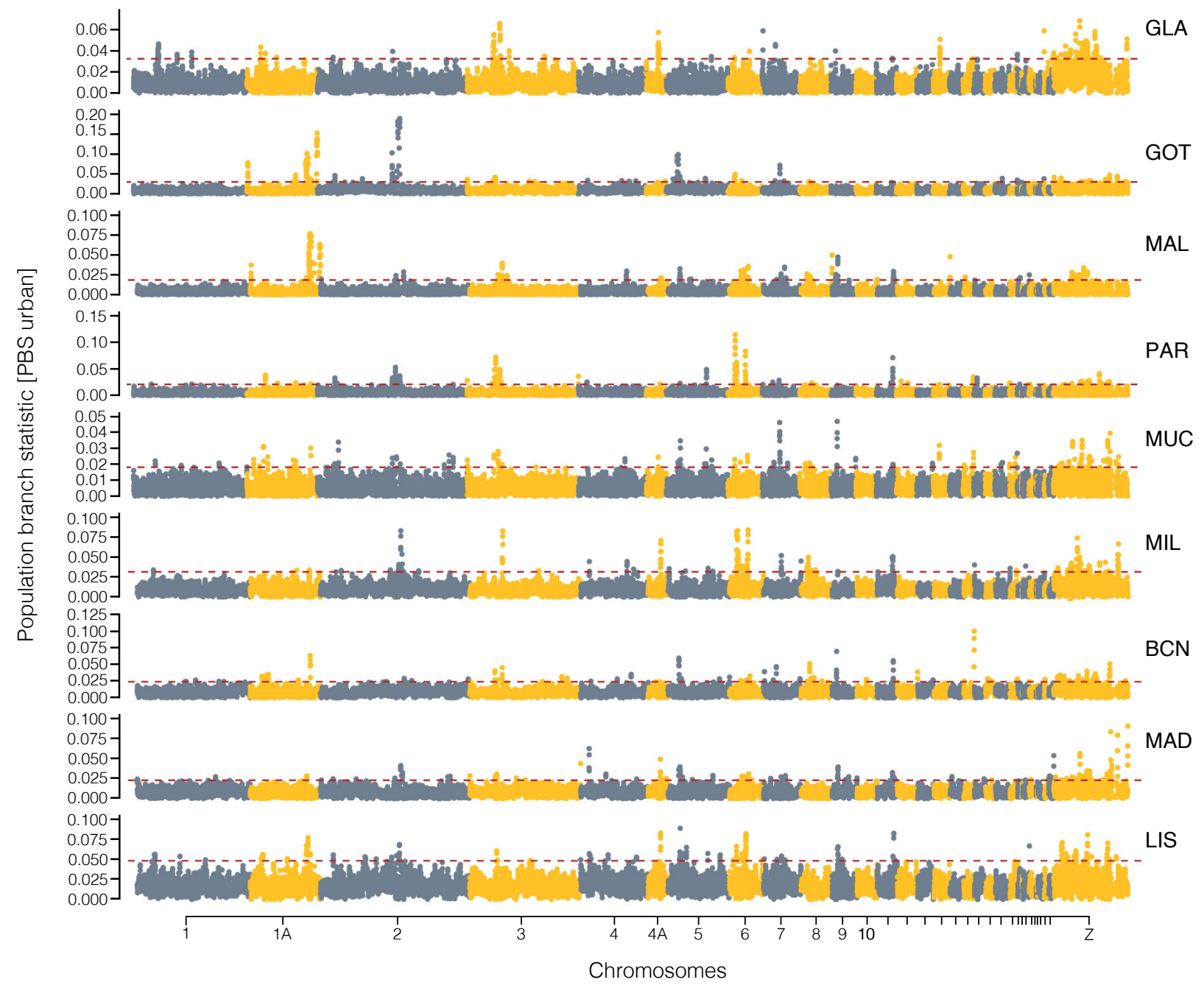

Fig. 3. Shared signals of selection across European urban great tit populations. Population branch statistics $(P B S)$ across the genome for each population in $200 \mathrm{~kb}$ sliding windows with $50 \mathrm{~kb}$ steps. Windows above the $99^{\text {th }}$ percentile of the empirical $P B S$ distribution (red dashed line) were selected as putatively under selection in urban populations. Only positive values were plotted here for visualisation 
purposes. Full distributions can be found in Fig. S7 and S8. BCN: Barcelona; GLA: Glasgow; GOT: Gothenburg; LIS: Lisbon; MAD: Madrid; MAL: Malmö; MIL: Milan; MUC: Munich; PAR: Paris.

To complement the above analyses and to strengthen the inference of urban selective sweeps under divergent selection, we also used haplotype-based statistics, which are more robust to the effect of low recombination and linked selection (Tang, Thornton, and Stoneking 2007). We compared haplotype-homozygosity between each urban and its respective rural population (Rsb score) but only focused on absolute values as the ancestral states of haplotypes were not known (Meier et al. 2018). Weak Spearman pairwise-correlations of the haplotype-based $R s b$ scores for each SNP (Fig. S9 and S10) across localities indicate that selective sweeps are mostly locality-specific, as suggested by the weak correlations of $P B S$.

Nonetheless, we identified several genes with recurrent signals of selection across a broad geographical scale ( $>3$ urban populations). The $R s b$ statistics revealed signatures of selective sweeps in or close to 568 genes in more than three localities (4-8 urban populations), many of which were also detected using the PBS statistic ("candidate genes under selection"; Fig. 3d; Table S3). Comparing genes associated with signatures of selection by $P B S$ and $R s b$ statistics, we identified 79 genes that showed signs of selection in at least three populations for both summary statistic (Table S3). Of these, 4 genes were putatively under selection in more than half of all urban populations ( $>5$ populations, maximum of 6 ) based on $P B S$ scores, and also showed signs of selection in 5 to 8 populations (median $=5 \pm 1.69$ s.d.) based on $R s b$ scores (Table S3). The recurrent signals of selection on these genes suggest the existence of genetic parallelism in the underlying response to urbanisation at the gene level and indicate that these genes might potentially be strong candidates in the adaptation to urban habitats.

\section{Evolutionary drivers of genetic divergence and signatures $f$ selection}

To test if signatures of selection were caused by divergent selection, or alternatively by background selection in low recombination regions (Burri 2017), we evaluated the correlation of signatures of selection with patterns of linkage disequilibrium (LD). First, we tested the correlation between genetic diversity (LD measured as $\mathrm{r}^{2}$ and summarized in $200 \mathrm{~kb}$ sliding windows with $50 \mathrm{~kb}$ steps per population and summarised as PC1 across all urban populations) with measures of selection ( $P B S$ and $R s b$ summarised as PC1 across populations). Overall, the 
correlation between genetic differentiation $(P B S-P C 1)$ and genetic diversity in urban populations $(L D-P C 1)$ was relatively weak but significant $\left(\mathrm{R}^{2}=-0.002 ; \mathrm{P}=9.7 \times 10^{-11}\right)$, with low diversity regions (high LD) showing weaker signatures of selection, i.e. low $P B S$ scores (Fig. 4a-c). This suggests that broad signatures of selection in urban habitats, positive $P B S-P C 1$, are likely not caused by background selection in low recombination regions but rather divergent selection (Burri et al. 2015). We detected similar correlation patterns in most populations when we assessed the correlation of urban genetic diversity $(L D-P C 1)$ with $P B S$ scores by locality, i.e. each independent city, rather than across all populations together (Fig. S11). However, in some localities, such as in Malmö (MAL) and Gothenburg (GOT), we detected a positive correlation between selection and genetic diversity $(P B S \sim L D-P C 1)$, suggesting the existence of spatially varying impacts of background selection on the genomic landscape of differentiation in great tits (Fig. S11). Similarly, the correlation between haplotype-homozygosity (Rsb-PCl) and genetic diversity $(L D-P C 1)$ was very weak but significant (all chromosomes: $\mathrm{R}^{2}=0.0115 ; \mathrm{P}<2.2 \times 10^{-}$ ${ }^{16}$, without $\mathrm{Z}$ chromosome: $\mathrm{R}^{2}=0.0021 ; \mathrm{P}=6.9 \times 10^{-10}$ ), with outlier windows not being strongly associated with low diversity regions (high LD) (Fig. 4d). This is expected, as the comparative nature of the haplotype-based $R s b$ score accounts for overall reduced diversity in shared lowrecombination regions. Nonetheless, detailed recombination rate analyses and whole-genome data will be needed in the future to thoroughly understand the effect of recombination, linked selection and divergent selection on the landscape of divergence in European great tits.

Furthermore, we assessed patterns of linkage disequilibrium around individual “candidate genes under selection” by locality (Figs. 4a and b). Low recombination regions or shared selective sweeps in urban and rural individuals would result in reduced genetic diversity in all populations (i.e. both urban and rural), as the recombination landscape in songbirds is generally highly conserved (Burri et al. 2015; Delmore et al. 2018). Yet, LD patterns around candidate genes differed strongly between chromosomes, suggesting effects of different evolutionary drivers (Fig. 4a and b), with most candidate regions not being located inside conserved low diversity regions (strongly correlated LD peaks) that might be indicative of a low recombination region. However, we found that one shared candidate gene $(C D H 18)$ that showed signatures of selection in five populations based on $R s b$ and $P B S$, was located inside a highly conserved LD peak on chromosome 2 (Fig. 4a, Table S3). This location potentially represents the centromere, and thus we cannot fully exclude the impact of background selection on the 
repeated signature of selection. However, the fact that $C D H 18$ only seems to show signatures of selection in urban populations but not rural populations suggests a role of divergent selection and adaptation to urban habitats.

a

LD [r2]

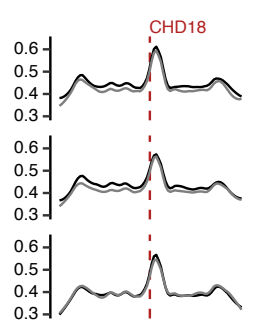

ح

ר.

0.6
0.5
0.4
0.4

ح

o.5

(150

Position chr. 2

C

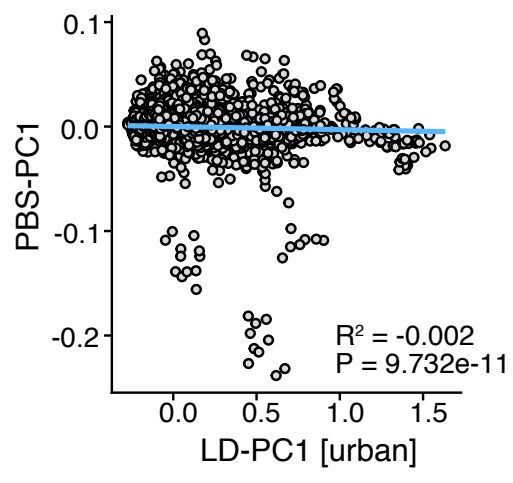

b
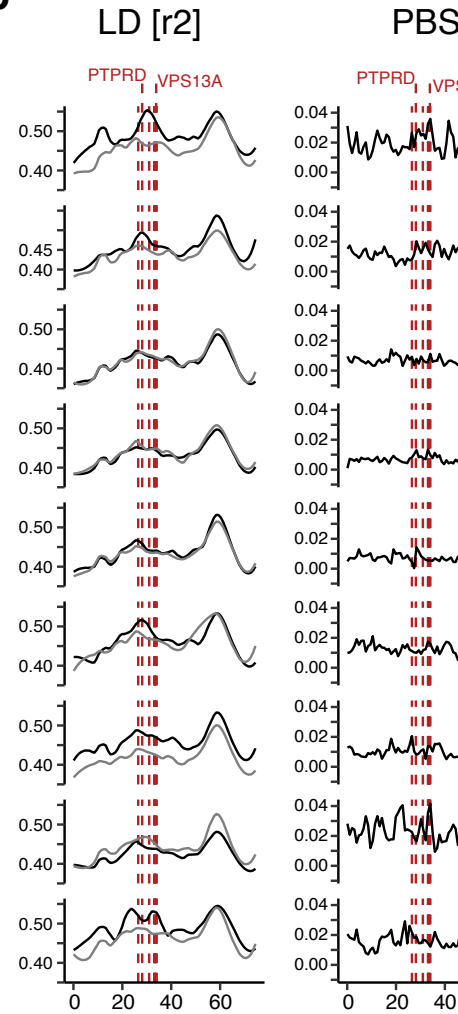

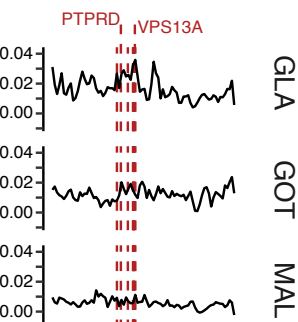

0.00

(111

$0.04-1$

0.00- unvillinmmym ¿

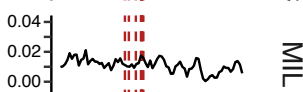

III

$0.00-2$ -

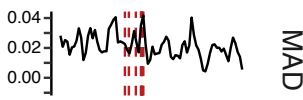

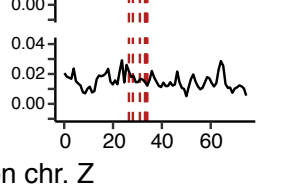

$$
\text { D }
$$

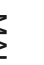

男

๖ุ

$\geqq$

吕

対

$\frac{5}{\omega}$

d

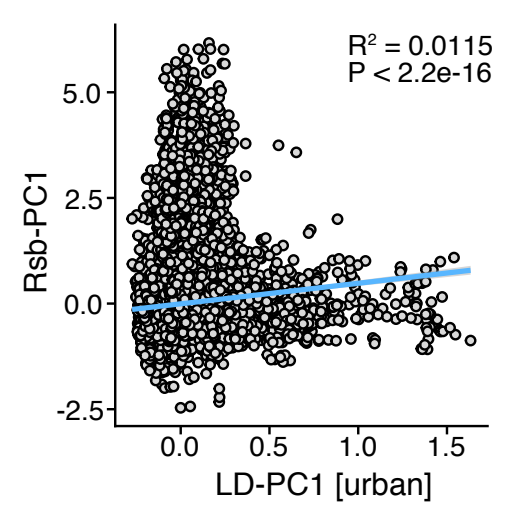

Fig. 4. Signatures of selection and LD. a, b Patterns of LD and $P B S$ around candidate genes associated with urbanisation on chromosome 2 and the $\mathrm{Z}$ chromosome. The positions of candidate genes are highlighted by red dashed lines. Patterns of LD for urban populations are shown in black and those for rural populations in grey. c, Correlation between $P B S-P C 1$ and $L D-P C 1$ (for urban populations) based on 
$200 \mathrm{~kb}$ sliding windows. d, Correlation between Rsb-PC1 and LD-PC1 (urban) based on the same $200 \mathrm{~kb}$ sliding windows. Note that windows with high $R s b$ values but low values of $L D-P C l$ are located on the $\mathrm{Z}$ chromosome. See text for details. BCN: Barcelona; GLA: Glasgow; GOT: Gothenburg; LIS: Lisbon; MAD: Madrid; MAL: Malmö; MIL: Milan; MUC: Munich; PAR: Paris.

Furthermore, LD was increased chromosome-wide in some urban populations compared to rural populations (e.g. chromosome Z, Fig. 4b), potentially suggesting an overall reduced genetic diversity and increased drift on those chromosomes. Nonetheless, local LD-peaks were still present around the "candidate genes under selection" in urban populations compared to rural populations (e.g. genes PTPRD and VPS13A in chromosome Z in Glasgow and Barcelona; Fig 4b, Fig. S12). The same genes also showed signs of selective sweeps in urban populations from localities without differences in baseline LD (e.g. chromosome Z in Milan; Figs. 4b), further supporting a scenario of divergent selection rather than increased drift due to locally reduced effective population size. Indeed, in birds, patterns of genetic differentiation at early stages of divergence, as presumably during colonisation of urban areas, have been found to be driven by selection rather than recombination (Burri 2017; Delmore et al. 2018). Thus, it is most likely that divergent selection rather than linked selection or drift explains the observed recurrent signals of selection in shared genes.

Interestingly, many of the SNPs putatively under selection were located on the Zchromosome, which in birds is the sex-chromosome in the homogametic sex (males) (Figs. 3 and $4 b)$. The strong urban association and selection signatures on this chromosome are in line with the "Fast-Z" evolution (Dean et al. 2015), which could be, at least partially, explained by a reduced recombination and effective population size on this particular chromosome. However, because many of these variants show similar allele frequency shifts between habitats across localities (Fig. 2f) and show signatures of selection in urban but not rural populations, recombination differences on their own cannot be the main driving factor of divergence in this particular chromosome.

\section{Convergent evolution of gene functions in response to urbanisation}

While the majority of genes showing signs of selection were unique to one or two cities, supporting the scenario of independent selective events, a significant proportion of genes were 
putatively under selection in multiple cities. Indeed, permutation analyses indicated that one would not expect, by chance, the same gene under selection in three or more cities $\left(\chi^{2}{ }_{1}=77.947\right.$, $\left.\mathrm{P}<2.2 \times 10^{-16}\right)$. However, 11 individual genes showed signs of selective sweeps in more than half of the studied urban populations (five to six) based on $P B S$, and also in more than three populations (three to eight) based on the $R s b$ statistic (Fig 5a, Table S3). Additionally, 107 genes were putatively under selection in three or four urban populations based on $P B S$ and also showed signs of selection based on $R s b$ in more than three populations (three to seven) (Table S3). These results corroborate and reinforce evidence for repeatability at the genomic level in the response to urbanisation between distant urban populations. Furthermore, 14 of these 79 genes putatively under selection were also associated with urbanisation-associated SNPs (see LFMM analyses).

Many of the genes associated with urbanisation and under recurrent selective sweeps in urban populations have previously been linked with behavioural divergence, suggesting adaptive phenotypic shifts related to behaviour. For instance, the PTPRD gene (chr. Z), which showed signs of selection in seven urban populations and was associated with urbanisation (Figs. 5b, Fig $\mathrm{S} 12$ ), encodes for the receptor-type tyrosine-protein phosphatase delta, an enzyme suggested to be involved in neural development of the hippocampus (Uetani et al. 2000), a brain region linked to spatial memory, bird navigation and flight performance (Mehlhorn, Haastert, and Rehkämper 2010; Gazda et al. 2018). The CDH18 gene (chr. 2) is part of a superfamily of membrane proteins involved in synaptic adhesion and was revealed as a candidate gene in phonological alterations in humans (Peter et al. 2016). Furthermore, VPS13A (chr. Z) gene variants in humans are linked to chorea-acanthocytosis (Ishida et al. 2009), a neurodegenerative disorder that affects movement and, this gene has recently shown to be associated with migratory behaviour in a songbird (Toews et al. 2019). 
a

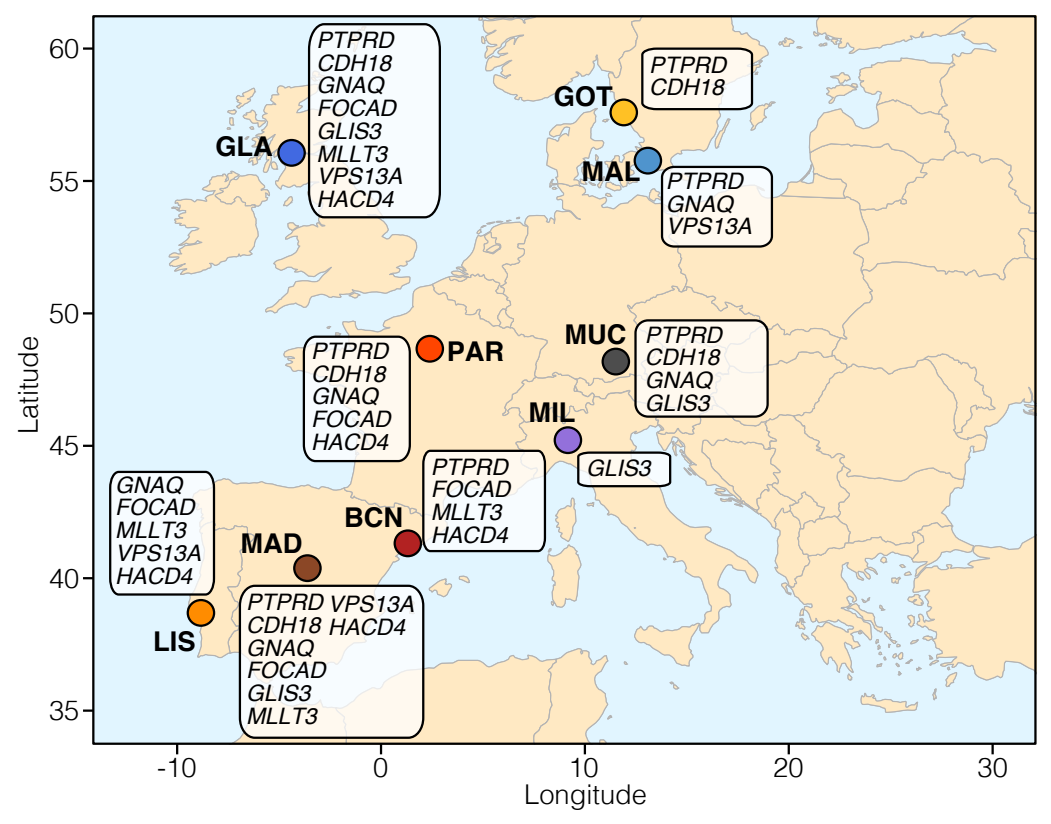

b

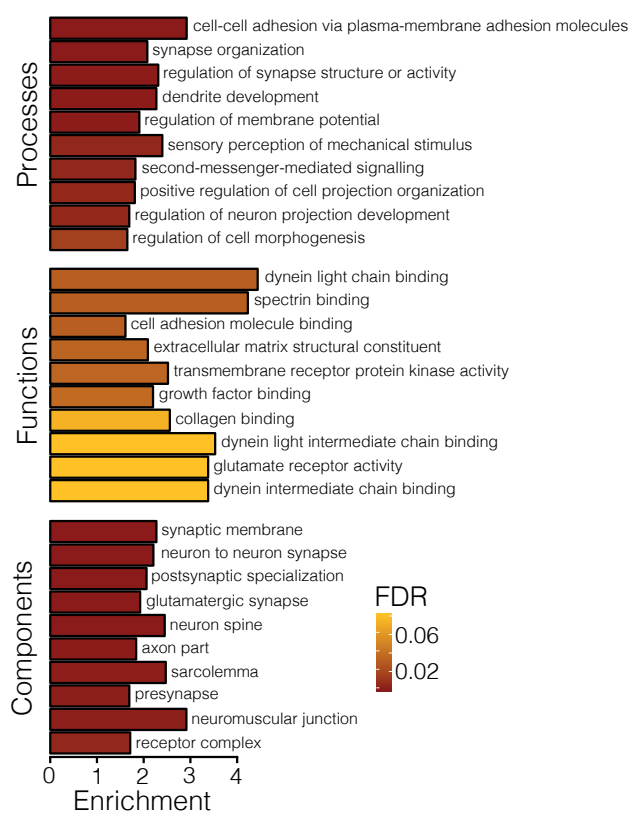

Fig. 5. Candidate genes and biological pathways associated with urbanisation. a, Map showing the spatial distribution of some top shared candidate genes putatively under selection in urban populations across Europe. b, Enrichment of associated genes in significantly enriched gene ontology (GO) groups by GO category in the LFMM and BayPass analysis (see Table S4 for a detailed list). The colour of bars represents the false-discovery rate (FDR) from the gene ontology overrepresentation analyses. BCN: Barcelona; GLA: Glasgow; GOT: Gothenburg; LIS: Lisbon; MAD: Madrid; MAL: Malmö; MIL: Milan; MUC: Munich; PAR: Paris.

Thus, our selection analyses suggest that natural selection repeatedly acts on behavioural traits and sensory and cognitive performance, all previously shown to be among the most widespread differences between urban and rural wildlife populations (Sih and Del Giudice 2012; Sol, Lapiedra, and González-Lagos 2013). These findings were furthermore supported by the GO terms analysis of the 2,758 urbanisation-associated SNPs (LFMM analysis), linked to 984 genes (1,501 SNPs in genic regions). Accordingly, most of the GO terms were related to neural functioning and development (e.g., GO:0016358, FDR $=4.30 \times 10^{-6}$ ), cell-adhesion (e.g., GO:0098742, FDR $=3.43 \times 10^{-6}$ ) and sensory perception (e.g., GO:0050954, FDR $=3.42 \times 10^{-3}$;

Fig. 4, Table S4). These GO terms were mainly clustered into two interacting networks, one related to sensory recognition and the other to neural development and cell adhesion (Fig. S13). These findings reinforce the previous idea on the importance of cognitive and behavioural changes as key responses to urbanisation in birds and in particular in great tits. Indeed, song structure and escape or distress behaviour have been previously shown to differ between urban 
and rural great tit populations across Europe (Senar et al. 2017; Slabbekoorn and den BoerVisser 2006; Møller and Ibáñez-Álamo 2012). Nonetheless, whether this is the result of a genetic response to selection or phenotypic plasticity is to a large extent still unknown. Only a few studies have previously explored the genetic patterns underlying urban adaptation in birds, finding evidence of divergence in behaviour-related genes at multiple European populations using either a candidate gene approach (J. C. Mueller et al. 2013) or a low-density SNP along transects within a city (Perrier et al. 2018). Furthermore, similar pathways showed divergence across three neighboring urban areas in a recently established urban-dwelling avian species in South America (Jakob C. Mueller et al. n.d.). Overall, our study supports these earlier observations regarding adaptive genetic changes in behavioural and neural development, suggesting that these processes play an important role in urban adaptation. Detailed functional genomic and phenotypic analyses are now needed to understand the role of these genes in the adaptive divergence of urban and rural great tits and other songbirds.

\section{Conclusions}

Our study demonstrates genetic signals of repeated local adaptation to urban habitats in a common songbird across multiple European cities. We found that a combination of parallel polygenic allele frequency shifts and recurrent but independent selective sweeps are associated with adaptation to urban environments. Our results strongly suggest that a few genes with known neural developmental and behavioural functions experienced recurrent but independent selective sweeps only in the urban populations. This suggests a strong consistency in the processes associated with urbanisation, despite the fact that underlying haplotypes are not shared. Thus, our study exemplifies repeated evolutionary adaptation to urban environments on a continental scale and highlights behavioural and neurosensory adjustments as important phenotypic adaptations in urban habitats. 


\section{Methods}

\section{Sample collection and DNA extraction}

During the years 2013-2015, 20 or more individual great tits were sampled at paired urbanrural sites from nine European cities (Figure 1a; Table S1). We sampled a total of 192 individuals (aged $>1$ year old) with 10-16 individuals per site (Table S1). Sexes were balanced between pairs (urban-rural) in the dataset (GLMM; pair: $\chi^{2}{ }_{1}=0.505, \mathrm{P}=0.477$ ). Each of the paired sampling sites (urban or rural, hereinafter populations) was sampled within the same season. Barcelona and Munich were sampled during winter period, however, in both cases only known birds (recaptures) were included in the study, thus, all birds can be considered resident. All urban populations were located within the city boundaries, the areas are characterized with significant proportion of human-built structures such as houses and roads with managed parks as the only green space. Rural populations were chosen to contrast the urban locations, regarding degree of urbanisation and were always natural/semi-natural forests and contained only a few isolated houses. Each urban and rural population were separated with a distance above the mean adult and natal dispersal distance of this species (i.e. see Table S1) (Paradis et al. 1998).

Blood samples (approx. $25 \mu \mathrm{l}$ ) were obtained either from the jugular or brachial vein and stored at $4{ }^{\circ} \mathrm{C}$ in ethanol or SET buffer and subsequently frozen at $-20^{\circ} \mathrm{C}$. In each case, procedures were identical for the paired rural and urban populations. DNA was extracted from approximately $5 \mu 1$ samples of red blood cells in $195 \mu$ l of phosphate-buffered saline using Macherey-Nagel NucleoSpin Blood Kits (Bethlehem, PA, USA) and following the manufacturer's instructions or manual salt extraction (ammonium acetate). The quantity and purity of the extracted genomic DNA was high and measured using a Nanodrop 2000 Spectrophotometer (Thermo Fisher Scientific) and Qubit 2.0 Fluorometer (Thermo Fisher Scientific).

\section{Urbanisation score}

To quantify the degree of urbanisation at each site we used the UrbanizationScore imageanalysis software, based on aerial images from Google Maps (Google Maps 2017) and following the methods previously described in different studies assessing the effect of urbanisation on wild bird populations (Seress et al. 2014). Briefly, each sampling site was represented by a $1 \mathrm{~km} \times 1$ 
$\mathrm{km}$ rectangular area around the capture locations. The content in each rectangle was evaluated dividing the image in $100 \mathrm{~m} \times 100 \mathrm{~m}$ cells and considering three land-cover characteristics in each: proportion of buildings, vegetation (including cultivated fields) and paved surfaces. The different land-cover measures obtained per site were used in a principal component analysis to estimate an urbanisation score variable $\left(\mathrm{PC}_{\mathrm{urb}}\right)$ for each of the urban or rural populations per locality, see Supplementary Table S1. The PC urb values were transformed to obtain negative values in the less urbanized and positive in the more urbanized sites. We used the average of the urbanisation estimates if birds were captured in more than one location within each site $(>2 \mathrm{~km}$ apart, mean \pm s.d.: $931.22 \pm 1,005.26 \mathrm{~m}$ ). All quantifications were done in triplicates by the same person (P.S.) and the estimates were highly repeatable, (intra class correlation coefficient, ICC = 0.993, 95\% CI $=0.997-0.987, \mathrm{P}<0.001)$.

\section{SNP genotyping}

All 192 individuals were successfully genotyped using a custom made Affymetrix $@$ great tit 650K SNP chip at Edinburgh Genomics (Edinburgh, United Kingdom). SNP calling was done following the "Best Practices Workflow" in the software Axiom Analysis Suite 1.1.0.616 (Affymetrix (C) and all the individuals passed the default quality control steps provided by the manufacturer (dish quality control values $>0.95$ ) and previous studies using the same SNP chip $(6,17)$. A total of 544,610 SNPs were then exported to a variant-calling format (VCF) and Plink and furthered filter and assigned to chromosomes using the great tit reference genome (GCA_001522545.2 Parus major v1.1; NCBI Annotation Release 101). 155 SNPs were not found in the new assembly and 17,927 SNPs were not in chromosomic regions; thus, these SNPs were removed from further analysis leaving a total of 526,528 SNPs.

\section{Genetic diversity and Population Structure}

We calculated the genome-wide genetic diversity as expected heterozygosity $\left(\mathrm{H}_{\mathrm{e}}\right)$ for each population using Plink 1.9 (Purcell et al. 2007), and tested if genetic diversity significantly differed between urban-rural populations from the same location using t-tests in $\mathrm{R}$ and overall across urban-rural pairings using a Wilcoxon rank sum test in $R$ (R Core Team 2018). Furthermore, we estimated pairwise F $_{\text {ST }}$ between all population-pairs (urban-rural per locality) 
using SNPRelate (Zheng et al. 2012). Mean average FST was computed across all comparisons after setting negative values to zero (Zheng et al. 2012).

For analyses of population structure, we pruned the SNP dataset based on linkage disequilibrium (LD) in Plink 1.9 using a variance inflation factor threshold of 2 ("-indep 505 2"), retaining 358,149 SNPs. Using this pruned and filtered dataset (314,350 SNPs), we performed a principal component analysis using SNPRelate (Zheng et al. 2012). Genetic ancestry analysis was done using the software package Admixture v.1.3 (Pickrell and Pritchard 2012) with $\mathrm{K}$ ranging from 2 to 18 and ten-fold cross-validation.

Additionally, we inferred a population tree based on allele-frequency co-variances using Treemix v.1.3 (Pickrell and Pritchard 2012), with blocks of 500 SNPs. In order to test for the potential of secondary gene flow across populations (cities) we fitted up to five migration edges and determined the best fitting tree based on the increase in maximum likelihood, variation explained, and on the significance of migration edges (Jacobs et al. 2020).

\section{Environment-associated SNPS}

We used two different approaches to identify SNPs associated with the degree of urbanisation. The degree of urbanisation was coded based on the "urbanisation score" to maintain gradual differences between rural and urban environments across the studied populations (see above). First, we used a univariate latent-factor linear mixed model implemented in LFMM for examining allele frequency - environment associations (Frichot et al. 2013). Based on the number of ancestry clusters (K) inferred with Admixture and the distribution of explained variation in the PCA (Fig. S2b), we ran LFMM with two and four latent factors, respectively. Each model was run 5 times for 10,000 iterations with a 5,000-iteration burn-in. We calculated the median z-score for each locus across all 10 runs and selected SNPs with a falsediscovery rate (FDR) below $1 \%$ to be associated with urbanisation. The results with two or four latent factors were highly concordant and the same candidate loci were recovered; thus, we only used the results obtained with four latent factors for further analyses.

Second, we analysed associations with urbanisation using the auxiliary covariate model implemented in BayPass v.2.1. (Gautier 2015). We estimated the allele-frequency - environment association for each SNP with the urbanisation score for each population accounting for population structure using a covariance matrix. We estimated the covariance matrix using the LD 
pruned SNP dataset in the core model using default parameters: 20 pilot runs of 1,000 iterations, a run length of 50,000 iterations, sampling every $25^{\text {th }}$ iteration, and a burn-in of 5,000 iterations. The resulting covariance matrix was used as input for five replicated runs of the auxiliary covariate model using the above settings. The strength of association is given in the test by estimated Bayes factor (measured in deciban; dB). We calculated the median Bayes Factor across all five replicated runs and considered all SNPs with a deciban unit $(\mathrm{dB})>20$ as urbanisation associated. This is the strictest criterion and is considered as "decisive evidence" for the association (Gautier 2015).

\section{Patterns of genetic differentiation $\left(F_{S T}, P B S\right)$}

To identify genomic regions distinguishing adjacent urban and rural great tits, we estimated the genetic differentiation (Weir \& Cockerham's F FT (Weir and Cockerham 1984)) for each urban and rural pair for each SNP using vcftools. We subsequently summarised and plotted FST values in $200 \mathrm{~kb}$ sliding windows with $50 \mathrm{~kb}$ steps using the windowscanr R-package. To identify genomic regions putatively under selection in urban but not rural populations, we also calculated the population branch statistic $(P B S)$ for each urban pair. We used the rural LIS (Lisbon) population as the outgroup as it is the genetically most distinct rural population, except for the $P B S$ estimation for the urban LIS population, for which we used the rural GLA (Glasgow) population as the outgroup. We used the following formula from Zhang et al. (Zhang et al. 2020): $\mathrm{PBS}_{\text {urb }}=\left(\mathrm{T}_{\text {urb-rur }}+\mathrm{T}_{\text {urb-out }}-\mathrm{T}_{\text {rur-out }}\right) / 2$, where $\mathrm{T}=-\log \left(1-\mathrm{F}_{\mathrm{ST}}\right) . \mathrm{T}_{\text {urb-rur }}$ is derived from $\mathrm{F}_{\mathrm{ST}}$ between adjacent rural and urban populations, $\mathrm{T}_{\text {urb-out }}$ from $\mathrm{F}_{\mathrm{ST}}$ between the focal urban and outgroup population, and $\mathrm{T}_{\text {rur-out }}$ from $\mathrm{F}_{\mathrm{ST}}$ between the rural and outgroup population. For visualisation purposes, only positive $P B S$ values, those showing putative signs of selection in the focal urban population, were plotted along the genome.

\section{Haplotype-based selection analysis}

To identify genomic regions showing signs of (incomplete) selective sweeps in urban-rural population pairs, we scanned the genome for regions of extended haplotype homozygosity (EHHS) in urban compared to rural populations. We firstly used fastPHASE (Scheet and Stephens 2006) to reconstruct haplotypes and impute missing data independently for each chromosome using the default parameters, except that each individual was classified by its 
population (“- $u$ " option). We used 10 random starts of the EM algorithm ("- $T$ " option) and 100 haplotypes (“- $H$ ” option). The fastPHASE output files were analysed using rehh 2.0 (Gautier, Klassmann, and Vitalis 2017). In addition, we used "rehh" to calculate $R s b$ statistics per focal SNP. The $R s b$ score is the standardized ratio of integrated EHHS (iES, which is a site-specific extended haplotype homozygosity) between two populations, is calculated using the following formula in rehh (Tang, Thornton, and Stoneking 2007; Gautier, Klassmann, and Vitalis 2017):

$$
R s b=\frac{L R i E S^{\text {Tang }}-\text { med }_{\text {LRiES }}{ }^{\text {Tang }}}{\sigma_{\text {LRiES }}{ }^{\text {Tang }}}
$$

, with LRiES ${ }^{\text {Tang }}$ representing the unstandardized log ratio of the $\mathrm{iES}^{\text {Tang }}$ (urban) and $\mathrm{iES}^{\text {Tang }}$ (rural) computed in the urban and rural populations (Gautier, Klassmann, and Vitalis 2017), and med $_{\text {LRiES,Tang }}$ and $\sigma_{\text {LRiES,Tang }}$ representing the median and standard deviation of LRiES ${ }^{\text {Tang }}$, respectively. This statistic measures the extent of haplotype homozygosity between two populations and follows the rationale that if a SNP is under selection in one population compared to the other, the region around this locus will show an unusually high level of haplotype homozygosity compared to the neutral distribution. As the we don't know the ancestral and derived state of each SNP, we focused on the absolute $R s b$ values. In accordance to Gautier and colleagues (Gautier, Klassmann, and Vitalis 2017), significant genomic regions were selected based on a threshold of $|R s b| \geq 4$. Because recombination rates can be assumed to be conserved between closely-related urban and rural populations, the cross-population comparative nature of the $R s b$ statistic provides an internal control that cancels out the effect of heterogeneous recombination across the genome (Tang, Thornton, and Stoneking 2007).

To determine the repeatability of selection across urban-centres, we implemented a resampling approach to assess the likelihood of genes showing signs of selection in two, three, four or more populations. We resampled with replacement $\mathrm{n}$ genes $(\mathrm{n}=$ number of genes with signatures of selection in each urban population) for each population from the list of all SNPlinked genes using the resample function in $R$, assessed the amount of overlap between populations (from two to 8 populations) and repeated the sampling 100,000 times for each comparison. We then calculated the mean and 95\% confidence interval (CI) for each comparison and compared the number of observed shared candidate genes to the expected number of 
candidate genes. The expected number of genes showing signs of selection in three or more populations was zero, thus we focused on genes showing signs of selection $(R s b, P B S)$ in three or more populations. This highlights the fact that it is unlikely that genes repeatedly show signs of selection in three or more urban populations by chance alone.

\section{Parallelism analyses of urbanisation-associated SNPS}

To determine the explanatory power of urbanisation associated SNPs and parallelism in allele frequency changes across populations, we performed a principal component analysis based on different candidate subsets, $i$ ) all LFMM candidate SNPs (PC between the LFMM and BayPass analyses ("Core urbanisation SNPs", $\mathrm{PC}_{\mathrm{GWAS}}$, see above), using SNPrelate. Furthermore, we ran a univariate linear model for each of the first three principal components (i.e. "PC $(\mathrm{PC} 1) \sim$ Habitat + Locality + Habitat $\times$ Locality") to quantitatively test the effect of parallel (significant "Habitat" effect, urban-rural) and non-parallel (significant "Habitat $\times$ Locality") on allele-frequency changes across populations. We used the "EtaSq" function implemented in BaylorEdPsych (Beaujean and Beaujean, n.d.) to extract the effect sizes (partial $\eta^{2}$ ) for the model terms in each linear model.

\section{Patterns of linkage disequilibrium (LD) across the genome}

To estimate the impact of variation in recombination rate and linked selection in lowrecombination regions on patterns of divergence (i.e. regions showing signs of selective sweeps), we estimated patterns of LD $\left(\mathrm{r}^{2}\right)$ across the genome using Plink 1.9 (Purcell et al. 2007). We focused on long-distance LD by calculating LD for pairs of SNPs within 2,000 to 200,000bp of each other for each population (each rural and urban population per locality). To reduce the computational load, we randomly sampled $5 \%$ of the dataset for each locality and plotted them for chromosomes containing candidate genes showing signs of selective sweeps in four or more cities (chromosome 2, chromosome Z). Furthermore, to investigate more large-scale patterns of correlation between selection and LD, we estimated a PCA based on LD scores in $200 \mathrm{~kb}$ sliding windows (50 kb steps) and used PC1 (LD-PC1) as a proxy for the distribution-wide LD pattern across the genome. We then estimated the PCA for PBS and estimated the correlation between 
$L D-P C 1$ and $P B S-P C 1$. We also estimated the correlation between $L D-P C 1$ and $P B S$ by locality to investigate local differences. We performed the same analyses for sliding window Rs $b$ scores.

\section{Functional characterization of candidate SNP}

We obtained the gene annotations for all candidate SNPs from the great tit reference genome annotation (GCA_001522545.2 Parus_major1.1; NCBI Annotation Release 101). We used all genes containing SNPs associated with urbanisation (LFMM and BayPass) $(\mathrm{N}=1,501$ SNPs within genes). To analyse the enrichment of functional classes, we identified overrepresented gene ontologies (biological processes, molecular functions and cellular components) using the WebGestalt software tool (Wang et al. 2017). The gene background was set using annotated great tit genes (Annotation release 101) containing SNPs from the SNP chip and with H. sapiens orthologues. H. sapiens genes were used as a reference set as human genes are better annotated with GO terms than those of any avian system (e.g. chicken) (Bosse et al. 2017; Laine et al. 2016). We focused on non-redundant gene ontology (GO) terms to account for correlations across the GO graph topology and GO terms as implemented in WebGestalt (Wang et al. 2017). A FDR < 0.05 was used as a threshold for significantly enriched GO terms. Furthermore, we searched the public record for functions of individual candidate genes. We also used GOrilla (Eden et al. 2009) to visualize the connections of GO terms associated with LFMM candidate genes and Cytoscape v.3.6.1 (Shannon et al. 2003) to visualize the GO network and identify all enriched GO terms (biological processes, $\mathrm{P}<0.001$ ), including redundant terms.

Candidate genes associated with signatures of selection were those that were either overlapping with PBS outlier windows or within $100 \mathrm{~kb}$ up- or down-stream of a SNP with an absolute Rsb score above 4. Overlap between genomic regions/SNPs and genes was assessed using bedtools.

\section{References}

Alberti, Marina, Cristian Correa, John M. Marzluff, Andrew P. Hendry, Eric P. Palkovacs, Kiyoko M. Gotanda, Victoria M. Hunt, Travis M. Apgar, and Yuyu Zhou. 2017. 'Global Urban Signatures of Phenotypic Change in Animal and Plant Populations'. Proceedings of the National Academy of Sciences of the United States of America 114 (34): 8951-56. https://doi.org/10.1073/pnas.1606034114.

Beaujean, A. Alexander, and Maintainer A. Alexander Beaujean. n.d. 'Package "BaylorEdPsych".

Bosse, Mirte, Lewis G. Spurgin, Veronika N. Laine, Ella F. Cole, Josh A. Firth, Phillip Gienapp, Andrew G. Gosler, et al. 2017. 'Recent Natural Selection Causes Adaptive Evolution of an Avian Polygenic Trait'. Science (New York, N.Y.) 358 (6361): 365-68. https://doi.org/10.1126/science.aal3298. 
Bouwhuis, S., B. C. Sheldon, S. Verhulst, and A. Charmantier. 2009. 'Great Tits Growing Old: Selective Disappearance and the Partitioning of Senescence to Stages within the Breeding Cycle'. Proceedings. Biological Sciences 276 (1668): 2769-77. https://doi.org/10.1098/rspb.2009.0457.

Boyce, Mark S., and C. M. Perrins. 1987. 'Optimizing Great Tit Clutch Size in a Fluctuating Environment'. Ecology 68 (1): 142-53. https://doi.org/10.2307/1938814.

Burri, Reto. 2017. 'Interpreting Differentiation Landscapes in the Light of Long-Term Linked Selection'. Evolution Letters 1 (3): 118-31. https://doi.org/10.1002/evl3.14.

Burri, Reto, Alexander Nater, Takeshi Kawakami, Carina F. Mugal, Pall I. Olason, Linnea Smeds, Alexander Suh, et al. 2015. 'Linked Selection and Recombination Rate Variation Drive the Evolution of the Genomic Landscape of Differentiation across the Speciation Continuum of Ficedula Flycatchers'. Genome Research 25 (11): 1656-65. https://doi.org/10.1101/gr.196485.115.

Caizergues, Aude E., Arnaud Grégoire, and Anne Charmantier. 2018. 'Urban versus Forest Ecotypes Are Not Explained by Divergent Reproductive Selection'. Proceedings of the Royal Society B: Biological Sciences 285 (1882): 20180261. https://doi.org/10.1098/rspb.2018.0261.

Campbell-Staton, Shane C., Kristin M. Winchell, Nicolas C. Rochette, Jason Fredette, Inbar Maayan, Rena M. Schweizer, and Julian Catchen. 2020. 'Parallel Selection on Thermal Physiology Facilitates Repeated Adaptation of City Lizards to Urban Heat Islands'. Nature Ecology \& Evolution 4 (4): 652-58. https://doi.org/10.1038/s41559-020-1131-8.

Charmantier, Anne, Virginie Demeyrier, Marcel Lambrechts, Samuel Perret, and Arnaud Grégoire. 2017. 'Urbanization Is Associated with Divergence in Pace-of-Life in Great Tits'. Frontiers in Ecology and Evolution 5. https://doi.org/10.3389/fevo.2017.00053.

Charmantier, Anne, Robin H. McCleery, Lionel R. Cole, Chris Perrins, Loeske E. B. Kruuk, and Ben C. Sheldon. 2008. 'Adaptive Phenotypic Plasticity in Response to Climate Change in a Wild Bird Population'. Science 320 (5877): 800-803. https://doi.org/10.1126/science.1157174.

Dean, Rebecca, Peter W. Harrison, Alison E. Wright, Fabian Zimmer, and Judith E. Mank. 2015. 'Positive Selection Underlies Faster-Z Evolution of Gene Expression in Birds'. Molecular Biology and Evolution 32 (10): 2646-56. https://doi.org/10.1093/molbev/msv138.

Delmore, Kira E., Juan S. Lugo Ramos, Benjamin M. Van Doren, Max Lundberg, Staffan Bensch, Darren E. Irwin, and Miriam Liedvogel. 2018. 'Comparative Analysis Examining Patterns of Genomic Differentiation across Multiple Episodes of Population Divergence in Birds'. Evolution Letters 2 (2): 76-87. https://doi.org/10.1002/evl3.46.

Eden, Eran, Roy Navon, Israel Steinfeld, Doron Lipson, and Zohar Yakhini. 2009. 'GOrilla: A Tool for Discovery and Visualization of Enriched GO Terms in Ranked Gene Lists'. BMC Bioinformatics 10 (February): 48. https://doi.org/10.1186/1471-2105-10-48.

Frichot, Eric, Sean D. Schoville, Guillaume Bouchard, and Olivier François. 2013. 'Testing for Associations between Loci and Environmental Gradients Using Latent Factor Mixed Models'. Molecular Biology and Evolution 30 (7): 1687-99. https://doi.org/10.1093/molbev/mst063.

Gautier, Mathieu. 2015. 'Genome-Wide Scan for Adaptive Divergence and Association with Population-Specific Covariates'. Genetics 201 (4): 1555-79. https://doi.org/10.1534/genetics.115.181453.

Gautier, Mathieu, Alexander Klassmann, and Renaud Vitalis. 2017. 'Rehh 2.0: A Reimplementation of the R Package Rehh to Detect Positive Selection from Haplotype Structure'. Molecular Ecology Resources 17 (1): 78-90. https://doi.org/10.1111/1755-0998.12634.

Gazda, Malgorzata A., Pedro Andrade, Sandra Afonso, Jolita Dilyte, John P. Archer, Ricardo J. Lopes, Rui Faria, and Miguel Carneiro. 2018. 'Signatures of Selection on Standing Genetic Variation Underlie Athletic and Navigational Performance in Racing Pigeons'. Molecular Biology and Evolution 35 (5): 1176-89. https://doi.org/10.1093/molbev/msy030.

Gienapp, Phillip, Veronika N. Laine, A. C. Mateman, Kees van Oers, and Marcel E. Visser. 2017. 'EnvironmentDependent Genotype-Phenotype Associations in Avian Breeding Time'. Frontiers in Genetics 8: 102. https://doi.org/10.3389/fgene.2017.00102.

Harris, Stephen E., and Jason Munshi-South. 2017. 'Signatures of Positive Selection and Local Adaptation to Urbanization in White-Footed Mice (Peromyscus Leucopus)'. Molecular Ecology 26 (22): 6336-50. https://doi.org/10.1111/mec.14369.

Hendry, Andrew P., Thomas J. Farrugia, and Michael T. Kinnison. 2008. 'Human Influences on Rates of Phenotypic Change in Wild Animal Populations'. Molecular Ecology 17 (1): 20-29. https://doi.org/10.1111/j.1365294X.2007.03428.x. 
Hendry, Andrew P., Kiyoko M. Gotanda, and Erik I. Svensson. 2017. 'Human Influences on Evolution, and the Ecological and Societal Consequences'. Philosophical Transactions of the Royal Society of London. Series B, Biological Sciences 372 (1712). https://doi.org/10.1098/rstb.2016.0028.

Isaksson, C., J. Sturve, B. C. Almroth, and S. Andersson. 2009. 'The Impact of Urban Environment on Oxidative Damage (TBARS) and Antioxidant Systems in Lungs and Liver of Great Tits, Parus Major',. Environmental Research 109 (1): 46-50. https://doi.org/10.1016/j.envres.2008.10.006.

Ishida, Chiho, Takao Makifuchi, Shinji Saiki, Genjiro Hirose, and Masahito Yamada. 2009. 'A Neuropathological Study of Autosomal-Dominant Chorea-Acanthocytosis with a Mutation of VPS13A'. Acta Neuropathologica 117 (1): 85-94. https://doi.org/10.1007/s00401-008-0403-1.

Jacobs, Arne, Madeleine Carruthers, Andrey Yurchenko, Natalia V. Gordeeva, Sergey S. Alekseyev, Oliver Hooker, Jong S. Leong, et al. 2020. 'Parallelism in Eco-Morphology and Gene Expression despite Variable Evolutionary and Genomic Backgrounds in a Holarctic Fish'. PLOS Genetics 16 (4): e1008658. https://doi.org/10.1371/journal.pgen.1008658.

Johnson, Marc T. J., and Jason Munshi-South. 2017. 'Evolution of Life in Urban Environments'. Science (New York, N.Y.) 358 (6363). https://doi.org/10.1126/science.aam8327.

Kim, J. M., A. W. Santure, H. J. Barton, J. L. Quinn, E. F. Cole, M. E. Visser, B. C. Sheldon, M. A.M. Groenen, K. van Oers, and J. Slate. 2018. 'A High-Density SNP Chip for Genotyping Great Tit (Parus Major) Populations and Its Application to Studying the Genetic Architecture of Exploration Behaviour'. Molecular Ecology Resources 18 (4): 877-891. https://doi.org/10.1111/1755-0998.12778.

Krebs, John R. 1971. 'Territory and Breeding Density in the Great Tit, Parus Major L.' Ecology 52 (1): 2-22. https://doi.org/10.2307/1934734.

Laine, Veronika N., Toni I. Gossmann, Kyle M. Schachtschneider, Colin J. Garroway, Ole Madsen, Koen J.F. Verhoeven, Victor De Jager, et al. 2016. 'Evolutionary Signals of Selection on Cognition from the Great Tit Genome and Methylome'. Nature Communications 7: 1-9. https://doi.org/10.1038/ncomms10474.

Lemoine, Mélissa, Kay Lucek, Charles Perrier, Verena Saladin, Frank Adriaensen, Emilio Barba, Eduardo J. Belda, et al. 2016. 'Low but Contrasting Neutral Genetic Differentiation Shaped by Winter Temperature in European Great Tits'. Biological Journal of the Linnean Society 118 (3): 668-85. https://doi.org/10.1111/bij.12745.

Mehlhorn, Julia, Burkhard Haastert, and Gerd Rehkämper. 2010. 'Asymmetry of Different Brain Structures in Homing Pigeons with and without Navigational Experience'. The Journal of Experimental Biology 213 (Pt 13): 2219-24. https://doi.org/10.1242/jeb.043208.

Meier, Joana Isabel, David Alexander Marques, Catherine Elise Wagner, Laurent Excoffier, and Ole Seehausen. 2018. 'Genomics of Parallel Ecological Speciation in Lake Victoria Cichlids'. Molecular Biology and Evolution 35 (6): 1489-1506. https://doi.org/10.1093/molbev/msy051.

Møller, A. P., and J. D. Ibáñez-Álamo. 2012. 'Escape Behaviour of Birds Provides Evidence of Predation Being Involved in Urbanization'. Animal Behaviour 84 (2): 341-48. https://doi.org/10.1016/j.anbehav.2012.04.030.

Mueller, J. C., J. Partecke, B. J. Hatchwell, K. J. Gaston, and K. L. Evans. 2013. 'Candidate Gene Polymorphisms for Behavioural Adaptations during Urbanization in Blackbirds'. Molecular Ecology 22 (13): 3629-3637. https://doi.org/10.1111/mec.12288.

Mueller, Jakob C., Martina Carrete, Stefan Boerno, Heiner Kuhl, José L. Tella, and Bart Kempenaers. n.d. 'Genes Acting in Synapses and Neuron Projections Are Early Targets of Selection during Urban Colonization'. Molecular Ecology n/a (n/a). Accessed 21 April 2020. https://doi.org/10.1111/mec.15451.

Paradis, Emmanuel, Stephen R. Baillie, William J. Sutherland, and Richard D. Gregory. 1998. 'Patterns of Natal and Breeding Dispersal in Birds'. Journal of Animal Ecology 67 (4): 518-536. https://doi.org/10.1046/j.13652656.1998.00215.x.

Perrier, Charles, Ana Lozano del Campo, Marta Szulkin, Virginie Demeyrier, Arnaud Gregoire, and Anne Charmantier. 2018. 'Great Tits and the City: Distribution of Genomic Diversity and Gene-Environment Associations along an Urbanization Gradient'. Evolutionary Applications 11 (5): 593-613. https://doi.org/10.1111/eva.12580.

Peter, Beate, Ellen M. Wijsman, Alejandro Q. Nato Jr, University of Washington Center for Mendelian Genomics, Mark M. Matsushita, Kathy L. Chapman, Ian B. Stanaway, et al. 2016. 'Genetic Candidate Variants in Two Multigenerational Families with Childhood Apraxia of Speech'. PLOS ONE 11 (4): e0153864. https://doi.org/10.1371/journal.pone.0153864.

Pettifor, R. A., C. M. Perrins, and R. H. McCleery. 1988. 'Individual Optimization of Clutch Size in Great Tits'. Nature 336 (6195): 160-62. https://doi.org/10.1038/336160a0. 
Pickrell, Joseph K., and Jonathan K. Pritchard. 2012. 'Inference of Population Splits and Mixtures from GenomeWide Allele Frequency Data'. PLoS Genetics 8 (11): e1002967. https://doi.org/10.1371/journal.pgen.1002967.

Pritchard, Jonathan K., Joseph K. Pickrell, and Graham Coop. 2010. 'The Genetics of Human Adaptation: Hard Sweeps, Soft Sweeps, and Polygenic Adaptation'. Current Biology: CB 20 (4): R208-215. https://doi.org/10.1016/j.cub.2009.11.055.

Purcell, Shaun, Benjamin Neale, Kathe Todd-Brown, Lori Thomas, Manuel A. R. Ferreira, David Bender, Julian Maller, et al. 2007. 'PLINK: A Tool Set for Whole-Genome Association and Population-Based Linkage Analyses'. American Journal of Human Genetics 81 (3): 559-75. https://doi.org/10.1086/519795.

R Core Team. 2018. 'R: A Language and Environment for Statistical Computing.' R Foundation for Statistical Computing, Vienna, Austria.

Rivkin, L. Ruth, James S. Santangelo, Marina Alberti, Myla F. J. Aronson, Charlotte W. de Keyzer, Sarah E. Diamond, Marie-Josée Fortin, et al. 2019. 'A Roadmap for Urban Evolutionary Ecology'. Evolutionary Applications 12 (3): 384-98. https://doi.org/10.1111/eva.12734.

Salmón, P., J.F. Nilsson, H. Watson, S. Bensch, and C. Isaksson. 2017. 'Selective Disappearance of Great Tits with Short Telomeres in Urban Areas'. Proceedings of the Royal Society B: Biological Sciences 284 (1862). https://doi.org/10.1098/rspb.2017.1349.

Scheet, Paul, and Matthew Stephens. 2006. 'A Fast and Flexible Statistical Model for Large-Scale Population Genotype Data: Applications to Inferring Missing Genotypes and Haplotypic Phase’. American Journal of Human Genetics 78 (4): 629-44. https://doi.org/10.1086/502802.

Senar, Juan Carlos, Laszlo Z. Garamszegi, Vallo Tilgar, Clotilde Biard, Gregorio Moreno-Rueda, Pablo Salmón, J. M. Rivas, et al. 2017. 'Urban Great Tits (Parus Major) Show Higher Distress Calling and Pecking Rates than Rural Birds across Europe'. Frontiers in Ecology and Evolution 5. https://doi.org/10.3389/fevo.2017.00163.

Seress, Gábor, Ágnes Lipovits, Veronika Bókony, and László Czúni. 2014. 'Quantifying the Urban Gradient: A Practical Method for Broad Measurements'. Landscape and Urban Planning 131: 42-50. https://doi.org/10.1016/j.landurbplan.2014.07.010.

Shannon, Paul, Andrew Markiel, Owen Ozier, Nitin S. Baliga, Jonathan T. Wang, Daniel Ramage, Nada Amin, Benno Schwikowski, and Trey Ideker. 2003. 'Cytoscape: A Software Environment for Integrated Models of Biomolecular Interaction Networks'. Genome Research 13 (11): 2498-2504. https://doi.org/10.1101/gr.1239303.

Sih, Andrew, and Marco Del Giudice. 2012. 'Linking Behavioural Syndromes and Cognition: A Behavioural Ecology Perspective'. Philosophical Transactions of the Royal Society of London. Series B, Biological Sciences 367 (1603): 2762-72. https://doi.org/10.1098/rstb.2012.0216.

Slabbekoorn, Hans, and Ardie den Boer-Visser. 2006. 'Cities Change the Songs of Birds'. Current Biology 16 (23): 2326-31. https://doi.org/10.1016/j.cub.2006.10.008.

Sol, Daniel, Oriol Lapiedra, and Cesar González-Lagos. 2013. 'Behavioural Adjustments for a Life in the City'. Animal Behaviour, Including Special Section: Behavioural Plasticity and Evolution, 85 (5): 1101-12. https://doi.org/10.1016/j.anbehav.2013.01.023.

Sprau, Philipp, Alexia Mouchet, and Niels J. Dingemanse. 2017. 'Multidimensional Environmental Predictors of Variation in Avian Forest and City Life Histories'. Behavioral Ecology 28 (1): 59-68. https://doi.org/10.1093/beheco/arw130.

Spurgin, Lewis G., Mirte Bosse, Frank Adriaensen, Tamer Albayrak, Christos Barboutis, Eduardo Belda, Andrey Bushuev, et al. 2019. 'The Great Tit HapMap Project: A Continental-Scale Analysis of Genomic Variation in a Songbird'. BioRxiv, February, 561399. https://doi.org/10.1101/561399.

Tang, Kun, Kevin R. Thornton, and Mark Stoneking. 2007. 'A New Approach for Using Genome Scans to Detect Recent Positive Selection in the Human Genome'. PLoS Biology 5 (7): e171. https://doi.org/10.1371/journal.pbio.0050171.

Toews, David P. L., Scott A. Taylor, Henry M. Streby, Gunnar R. Kramer, and Irby J. Lovette. 2019. 'Selection on VPS13A Linked to Migration in a Songbird'. Proceedings of the National Academy of Sciences 116 (37): 18272-74. https://doi.org/10.1073/pnas.1909186116.

Uetani, Noriko, Kunio Kato, Hiro Ogura, Kazuya Mizuno, Kano Kawano, Katsuhiko Mikoshiba, Hidetaka Yakura, Masahide Asano, and Yoichiro Iwakura. 2000. 'Impaired Learning with Enhanced Hippocampal LongTerm Potentiation in PTP $\delta$-Deficient Mice'. The EMBO Journal 19 (12): 2775-85. https://doi.org/10.1093/emboj/19.12.2775. 
Wang, Jing, Suhas Vasaikar, Zhiao Shi, Michael Greer, and Bing Zhang. 2017. 'WebGestalt 2017: A More Comprehensive, Powerful, Flexible and Interactive Gene Set Enrichment Analysis Toolkit'. Nucleic Acids Research 45 (W1): W130-37. https://doi.org/10.1093/nar/gkx356.

Weir, B. S., and C. Clark Cockerham. 1984. 'Estimating F-Statistics for the Analysis of Population Structure'. Evolution 38 (6): 1358-70. https://doi.org/10.2307/2408641.

Yi, Xin, Yu Liang, Emilia Huerta-Sanchez, Xin Jin, Zha Xi Ping Cuo, John E. Pool, Xun Xu, et al. 2010. 'Sequencing of 50 Human Exomes Reveals Adaptation to High Altitude'. Science 329 (5987): 75-78. https://doi.org/10.1126/science.1190371.

Zhang, Shao-jie, Guo-Dong Wang, Pengcheng Ma, Liang-liang Zhang, Ting-Ting Yin, Yan-hu Liu, Newton O. Otecko, et al. 2020. 'Genomic Regions under Selection in the Feralization of the Dingoes'. Nature Communications 11 (1): 1-10. https://doi.org/10.1038/s41467-020-14515-6.

Zheng, Xiuwen, David Levine, Jess Shen, Stephanie M. Gogarten, Cathy Laurie, and Bruce S. Weir. 2012. 'A HighPerformance Computing Toolset for Relatedness and Principal Component Analysis of SNP Data'. Bioinformatics (Oxford, England) 28 (24): 3326-28. https://doi.org/10.1093/bioinformatics/bts606.

Acknowledgements: We are truly thankful to J. Pérez-Tris, J. Ignacio Aguirre de Miguel, M. Morganti, ringers from La Herreria and Lago di Pusiano, V. Encarnação, A. Mouchet and Pardal family. We also thank V.N. Laine, A. Herrera-Dueñas and A.C. Mateman for their help during data extraction and formatting and laboratory work and M. Bosse for comments on an early version of the manuscript. Funding: was provided by the Swedish Research Council C0361301 and Marie Curie Career Integration Grant FP7-CIG ID:322217 (to C.I.), Ministry of Economics and Competiveness (CGL-2016-79568-C3-3-P to J.C.S). Authors contribution: C.I. and P.S. conceived the study. P.S. collected and coordinated the sample collection with additions from C.B., N.D., D.M.D., B.H., J.C. and Ph.S. The data analysis was carried out by A.J. and P.S. with help from M.L., D.A. and M.E.V. C.I., A.J. and P.S. drafted the initial manuscript with input from all the other authors. Competing interest: all the authors declare not having any competing interest. 


\section{Supplementary Materials for}

\section{Repeated genomic signatures of adaptation to urbanisation in a songbird across Europe}

Pablo Salmón ${ }^{\dagger}$, Arne Jacobs $\dagger$, Dag Ahrén, Clotilde Biard, Niels J. Dingemanse, Davide Dominoni, Barbara Helm, Max Lundberg, Juan Carlos Senar, Philipp Sprau, Marcel Visser and Caroline Isaksson.

${ }^{\dagger}$ Both authors contributed equally

Correspondence to: Caroline.Isaksson@biol.lu.se 
bioRxiv preprint doi: https://doi.org/10.1101/2020 05.05.078568; this version posted May 7, 2020. The copyright holder for this preprint (which was not certified by peer review) is the author/funder, who has granted bioRxiv a license to display the preprint in perpetuity. It is made available under aCC-BY-ND 4.0 International license.
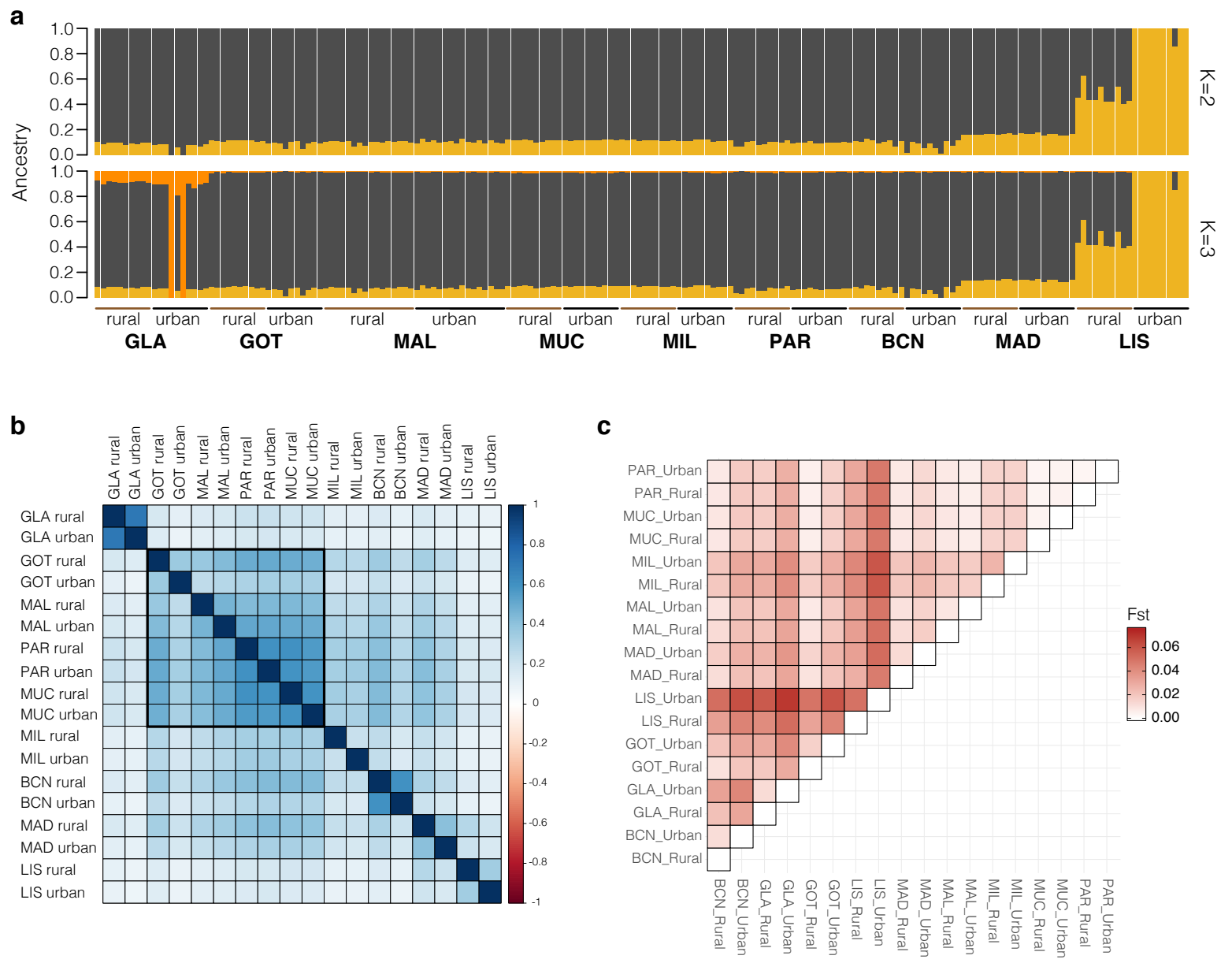

Fig. S1. Genetic ancestry in urban and rural European great tits. a, Genetic ancestry inferred with Admixture for $\mathrm{K}=2(\mathrm{CV}$-error $=0.615)$ and $\mathrm{K}=3(\mathrm{CV}$-error $=0.618)$, showing two main genetic clusters across Europe. b, Genetic correlation matrix showing genome-wide genetic similarities among all studied populations inferred using BayPass. The colour gradient indicates the strength of the correlation. c, Heatmap of pairwise $F_{S T}$ values between populations. BCN: Barcelona; GLA: Glasgow; GOT: Gothenburg; LIS: Lisbon; MAD: Madrid; MAL: Malmö; MIL: Milan; MUC: Munich; PAR: Paris. 
a
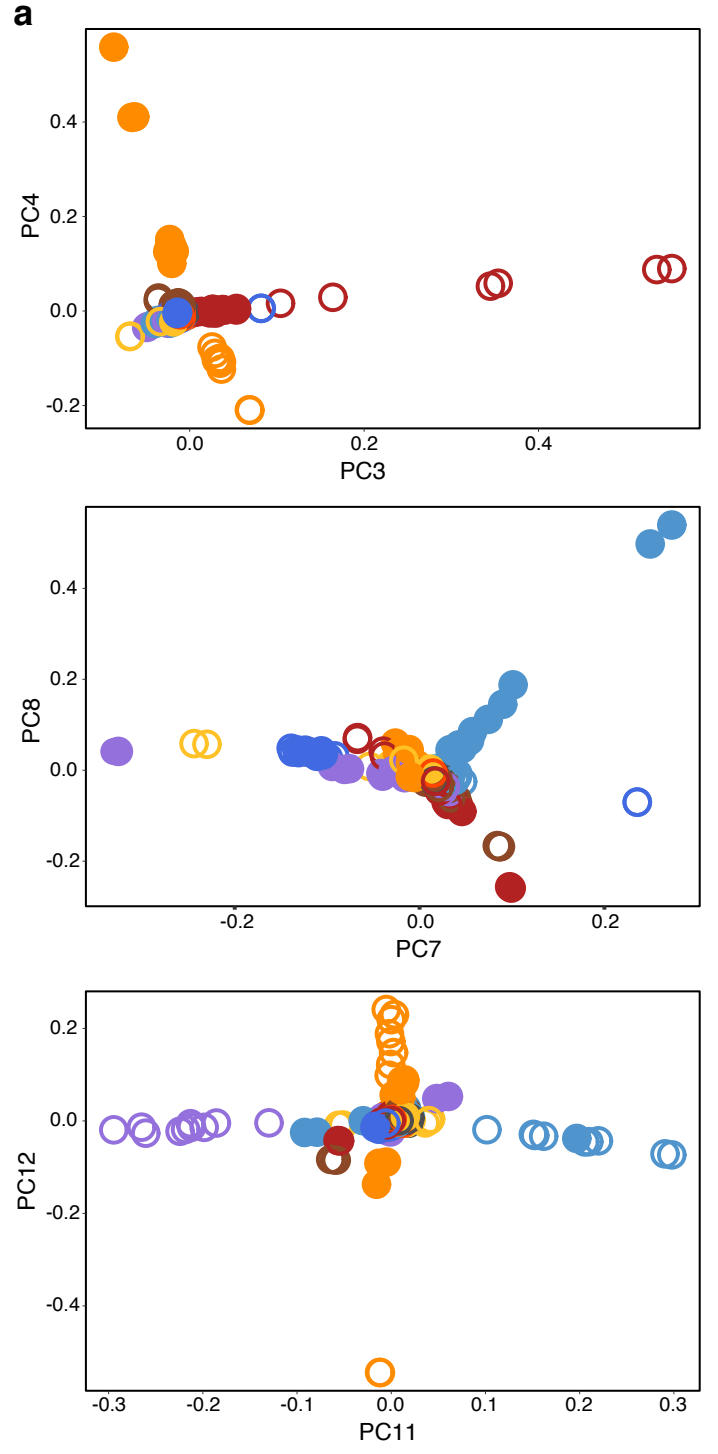
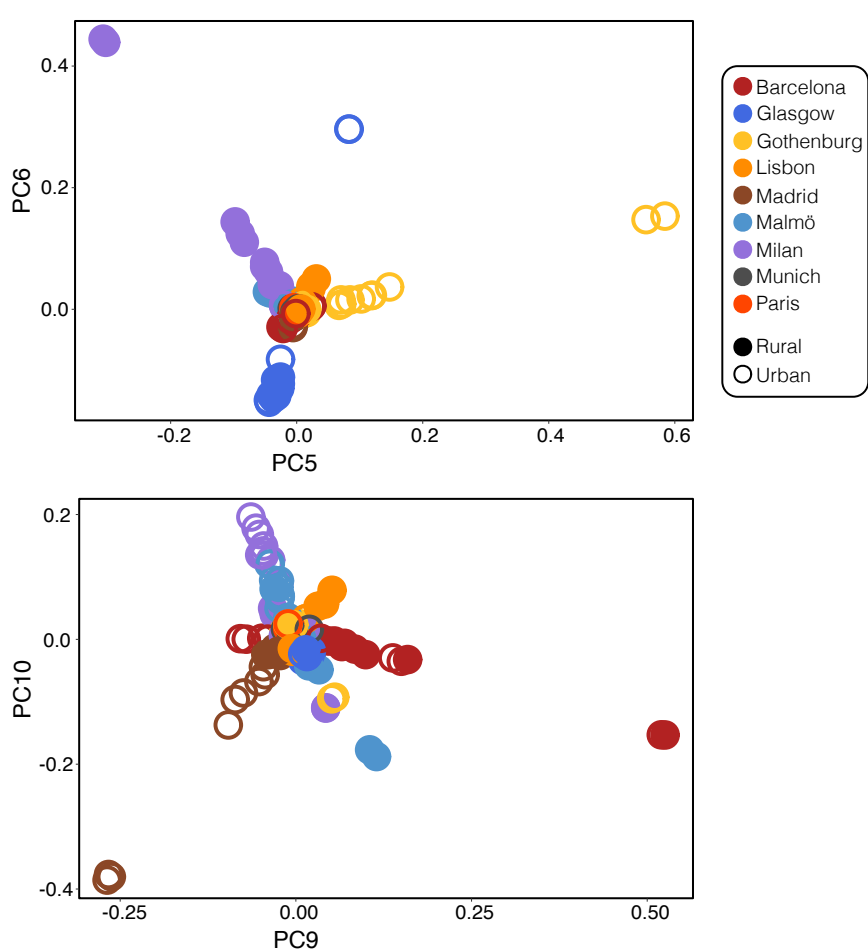

b

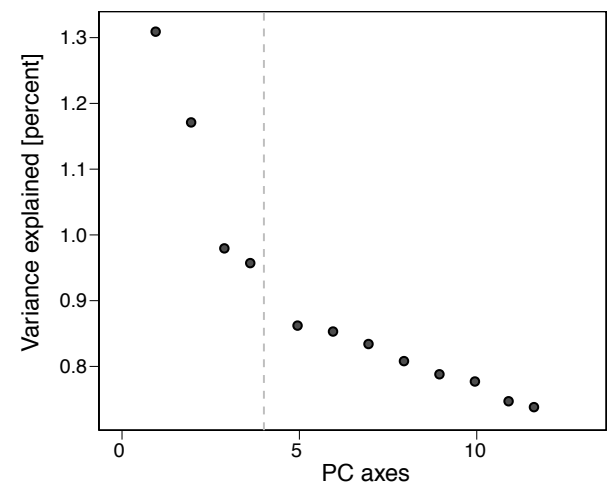

Fig. S2. Population structure of European great tits. a, Principal component plots displaying the divergence of great tit populations along eigenvectors 3-12 $\left(\mathrm{PC}_{\mathrm{Gen}}\right)$. b. Percentage of total variance explained by the first 12 principal components. The dashed grey line indicates the number of eigenvectors used for population structure correction in $L F M M$ based on the flattening of the distribution $(\mathrm{K}=2-4)$. 
a
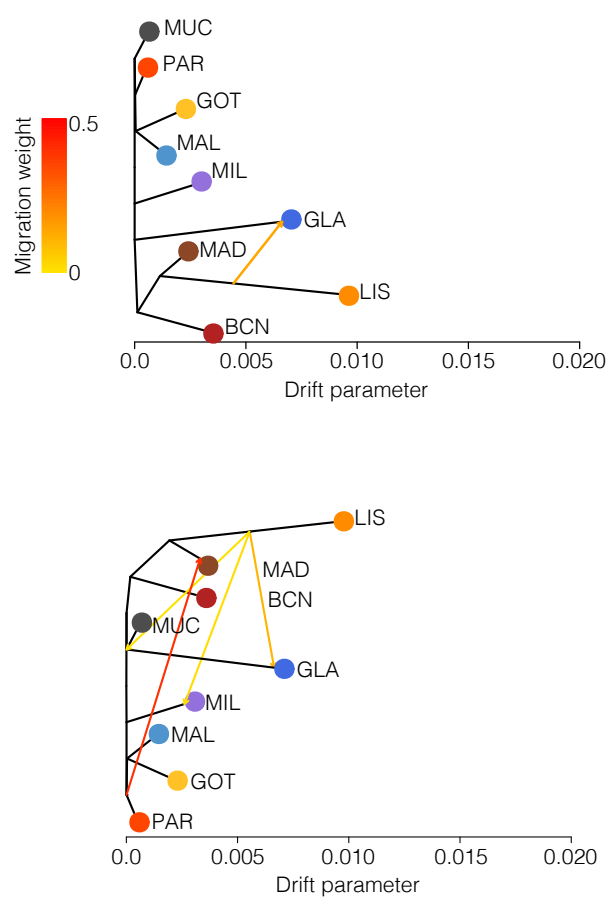
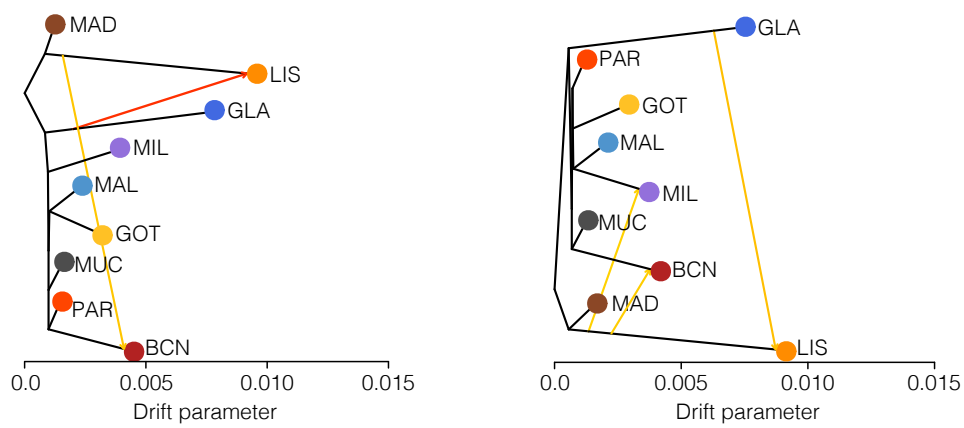

b
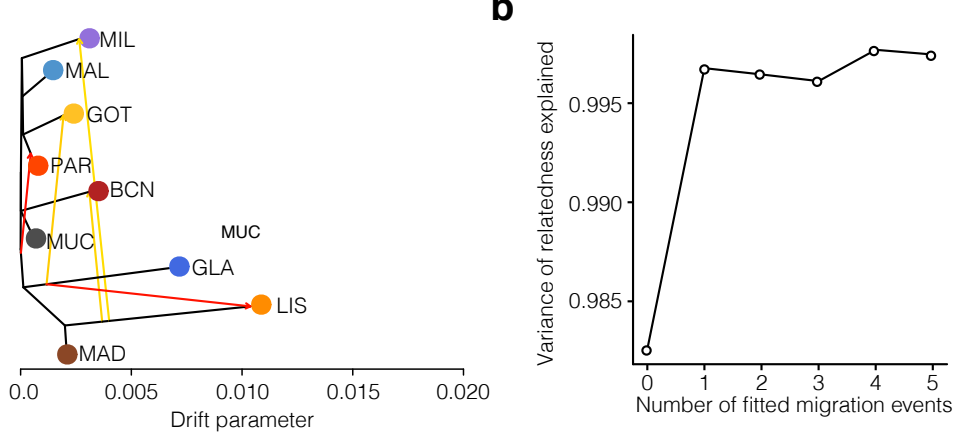

Fig. S3. Treemix maximum-likelihood trees. a, Allele frequency based unrooted maximum-likelihood Treemix trees including one, two, three, four and five migration edges. The colour of the arrows shows the migration weight for gene flow events between branches. b, Proportion of variance explained by models with zero to five migration edges. Note the reduced rate of increase after adding one migration event. 
a

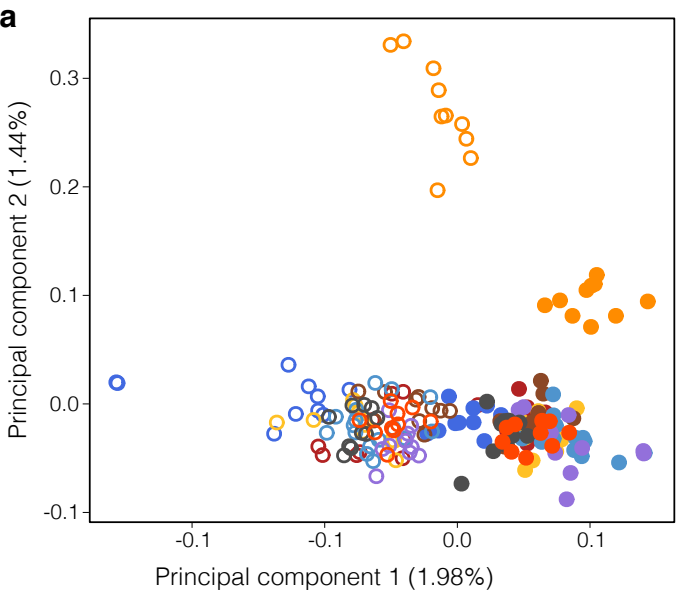

\section{b}

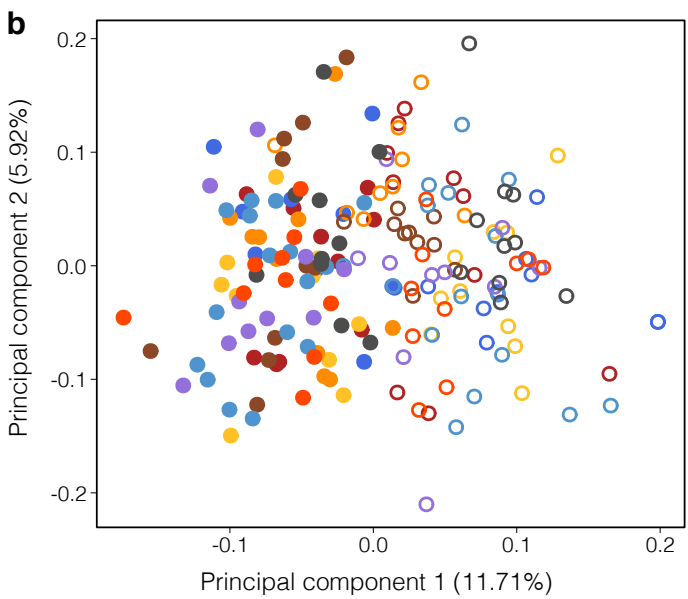

Barcelona Glasgow Gothenburg Lisbon Malmö Milan Munic - Paris - Rural OUrban

Fig. S4. PCA based on LFMM and "Core urbanisation SNPs". The first two principal component axis are shown (Percentage of total variance explained), based on a, $L F M M$ candidate SNPs $(\mathrm{N}=2,758)$ and $\mathrm{b}$, shared "core urbanisation SNPs" between LFMM and BayPass $(\mathrm{N}=34)$. 
bioRxiv preprint doi: https://doi.org/10.1101/2020.05.05.078568; this version posted May 7, 2020. The copyright holder for this preprint (which was not certified by peer review) is the author/funder, who has granted bioRxiv a license to display the preprint in perpetuity. It is made

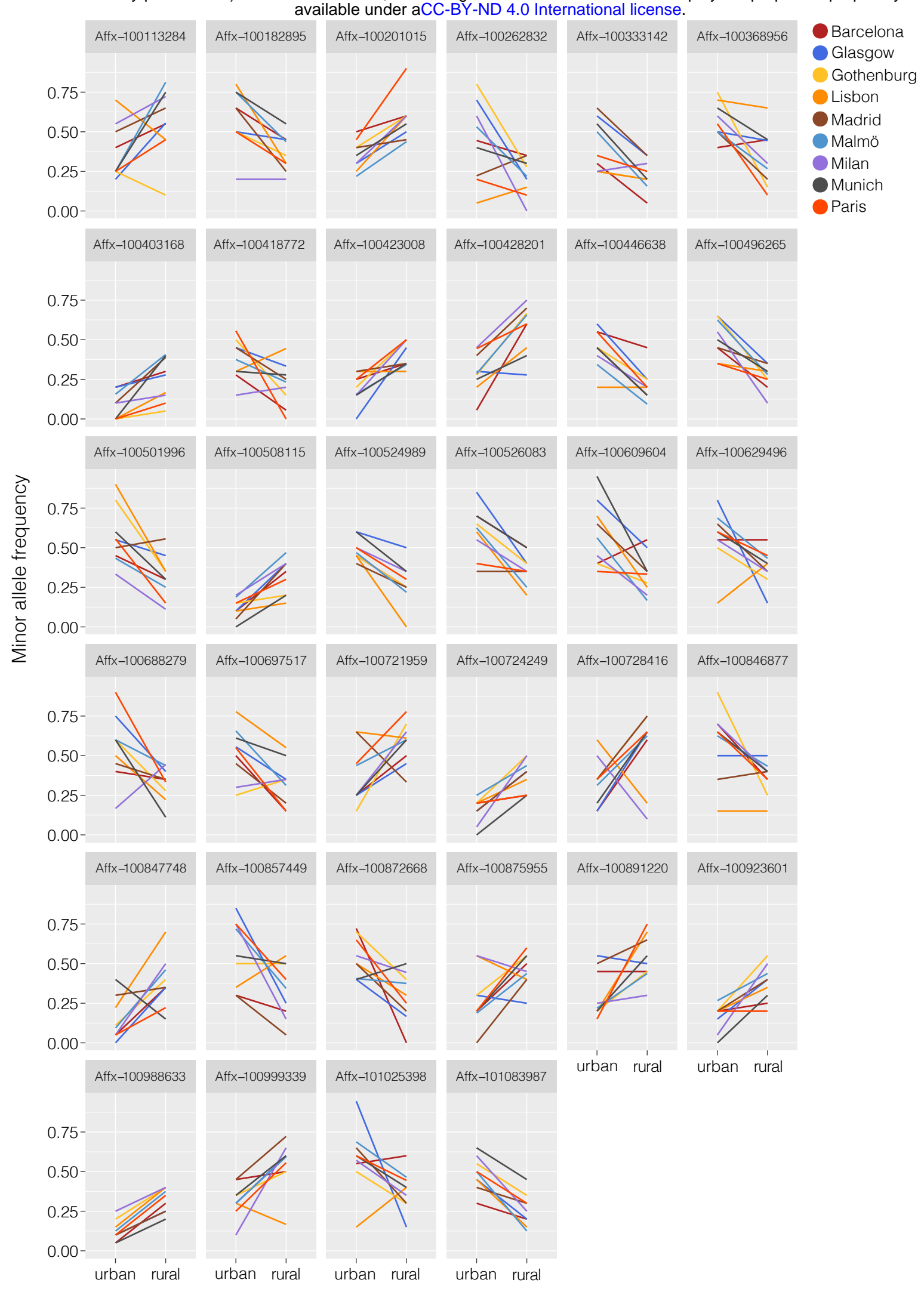

Fig. S5. Differences in allele frequency between urban and rural populations in the "core urbanisation SNPs". Minor allele frequency (MAF) trajectories between urban and rural populations for all "core urbanisation SNPs" $(\mathrm{N}=34)$. The Affx-number in the header shows the name for each SNP on the Affymetrix SNP-chip used for the genotyping of all individuals in this study. 

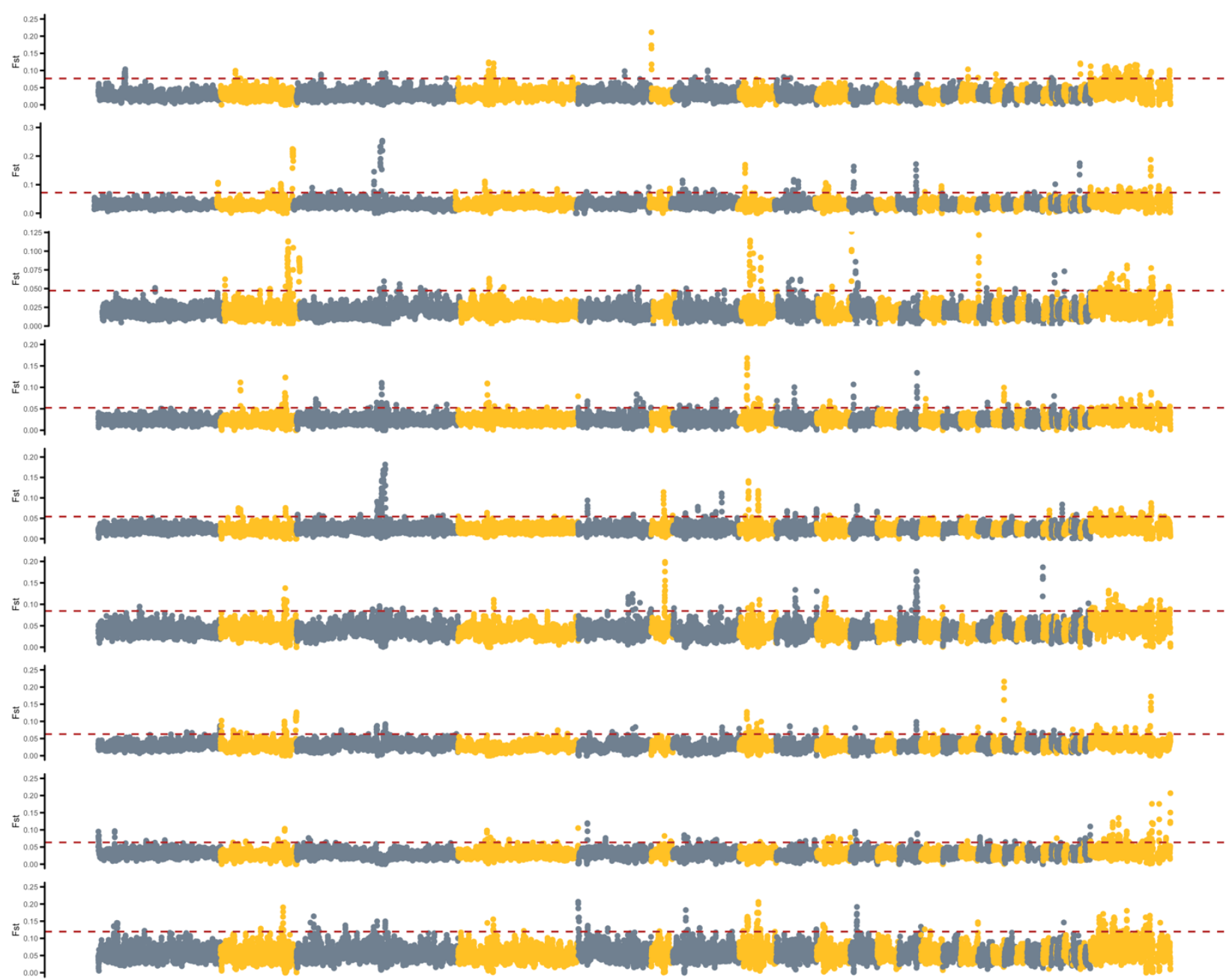

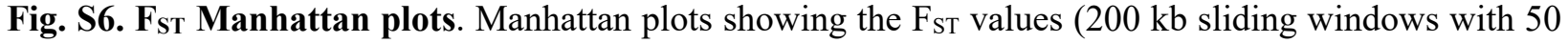
$\mathrm{kb}$ steps) between urban and rural individuals across the genome for each population pair (urban-centre). The red dashed line shows the $99^{\text {th }}$ percentile of the $\mathrm{F}_{\mathrm{ST}}$ distribution for each population pair. 


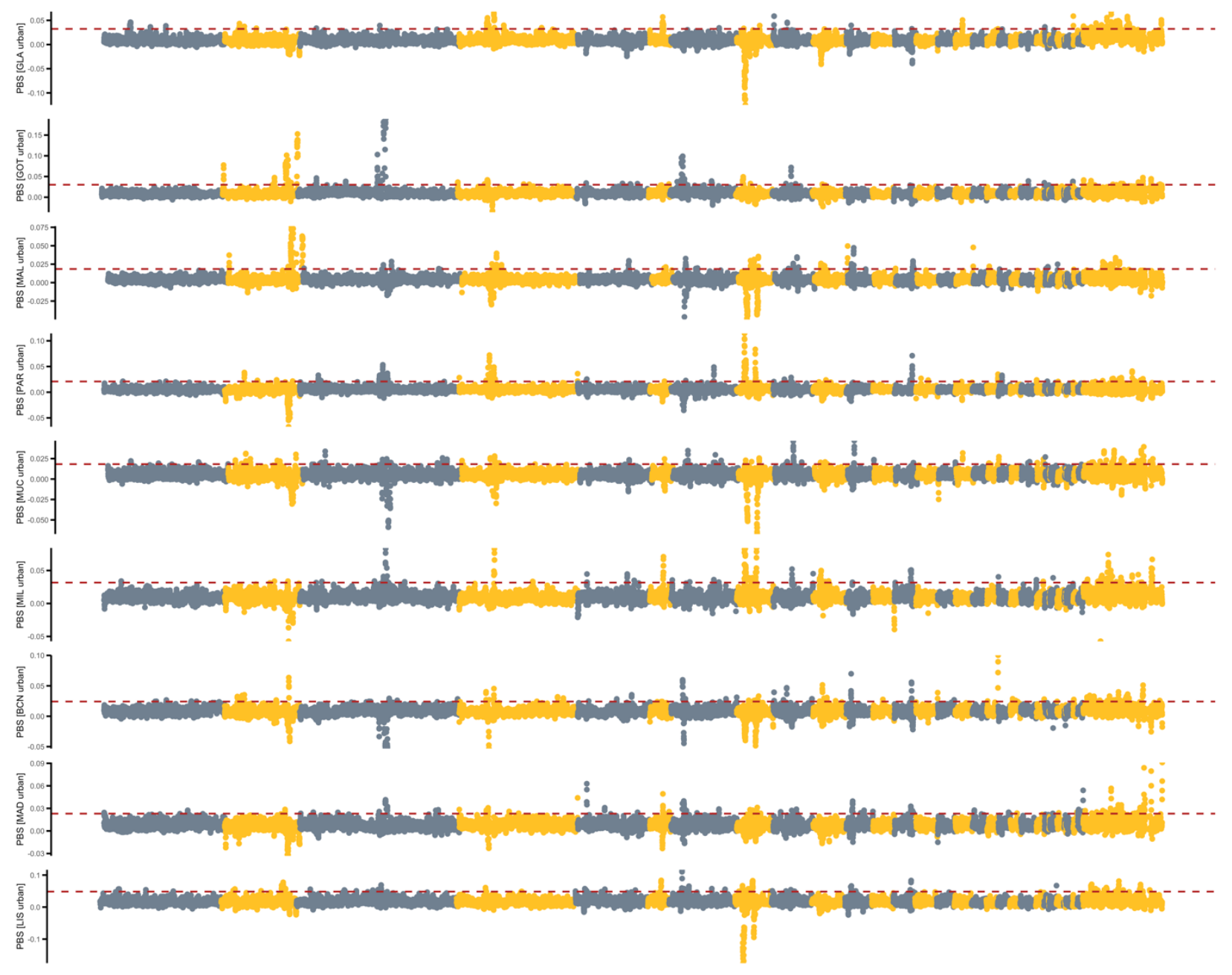

Fig. S7. PBS Manhattan plots with LIS rural as outgroup. Manhattan plots showing the window-based $P B S$ score (200 kb windows with $50 \mathrm{~kb}$ steps) between urban and rural individuals across the genome for each population pair (urban-centre). $P B S$ scores were calculated using the rural population from Lisbon as an outgroup., $P B S$ for Lisbon was calculated using GLA rural as the outgroup. The dashed lines show the $99^{\text {th }}$ percentile of the $P B S$ distribution. 

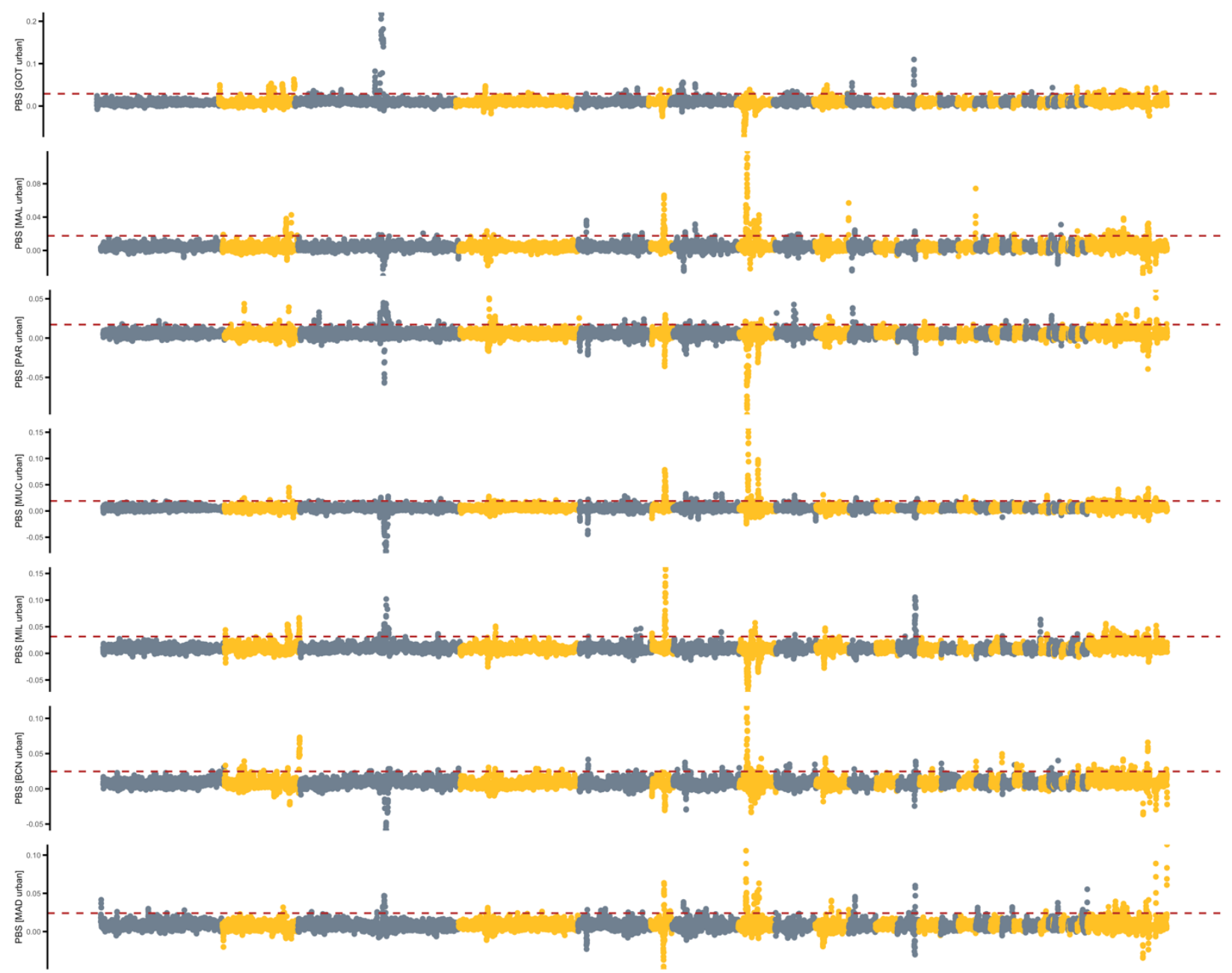

Fig. S8. PBS Manhattan plots with GLA rural as outgroup. Manhattan plots showing the window-based $P B S$ score (200 kb windows with $50 \mathrm{~kb}$ steps) between urban and rural individuals across the genome for each population pair (urban-centre). $P B S$ scores were calculated using the rural population from Glasgow as an outgroup. PBS scores are shown for all population pairs except Glasgow and Lisbon. The dashed lines show the $99^{\text {th }}$ percentile of the PBS distribution. 

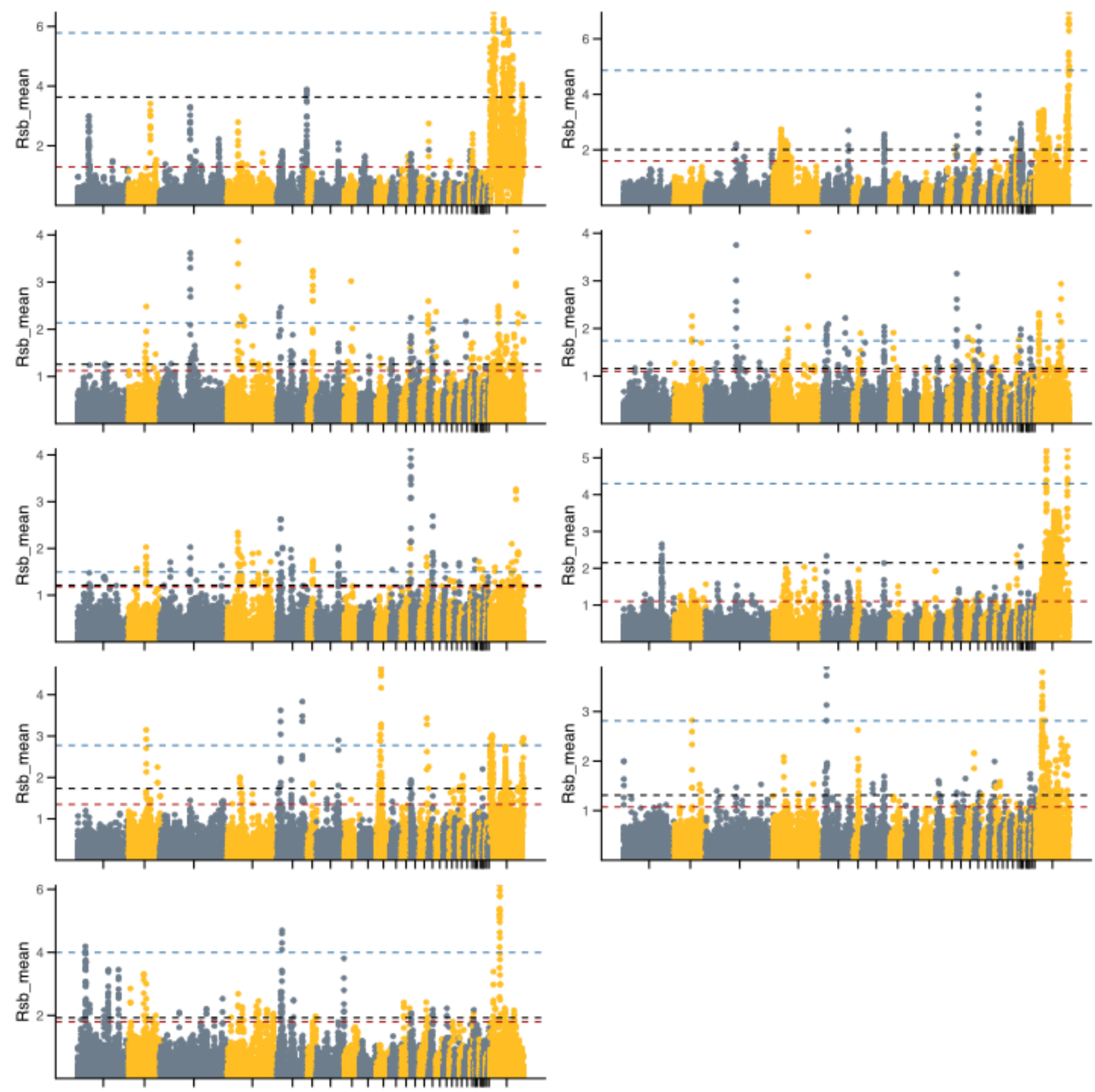

Fig. S9. Rsb Manhattan plots. Manhattan plots showing the absolute haplotype-based selection scores ( $R s b$ value in $200 \mathrm{~kb}$ windows with $50 \mathrm{~kb}$ steps) between urban and rural individuals across the genome for each population pair (urban-centre). The dashed lines show the $99^{\text {th }}$ percentile of the $R s b$ distribution for all chromosomes (black), only autosomes (red) and only the Z chromosome (blue). We selected outlier SNPs as those with SNP-based $R s b$ values above 4. 


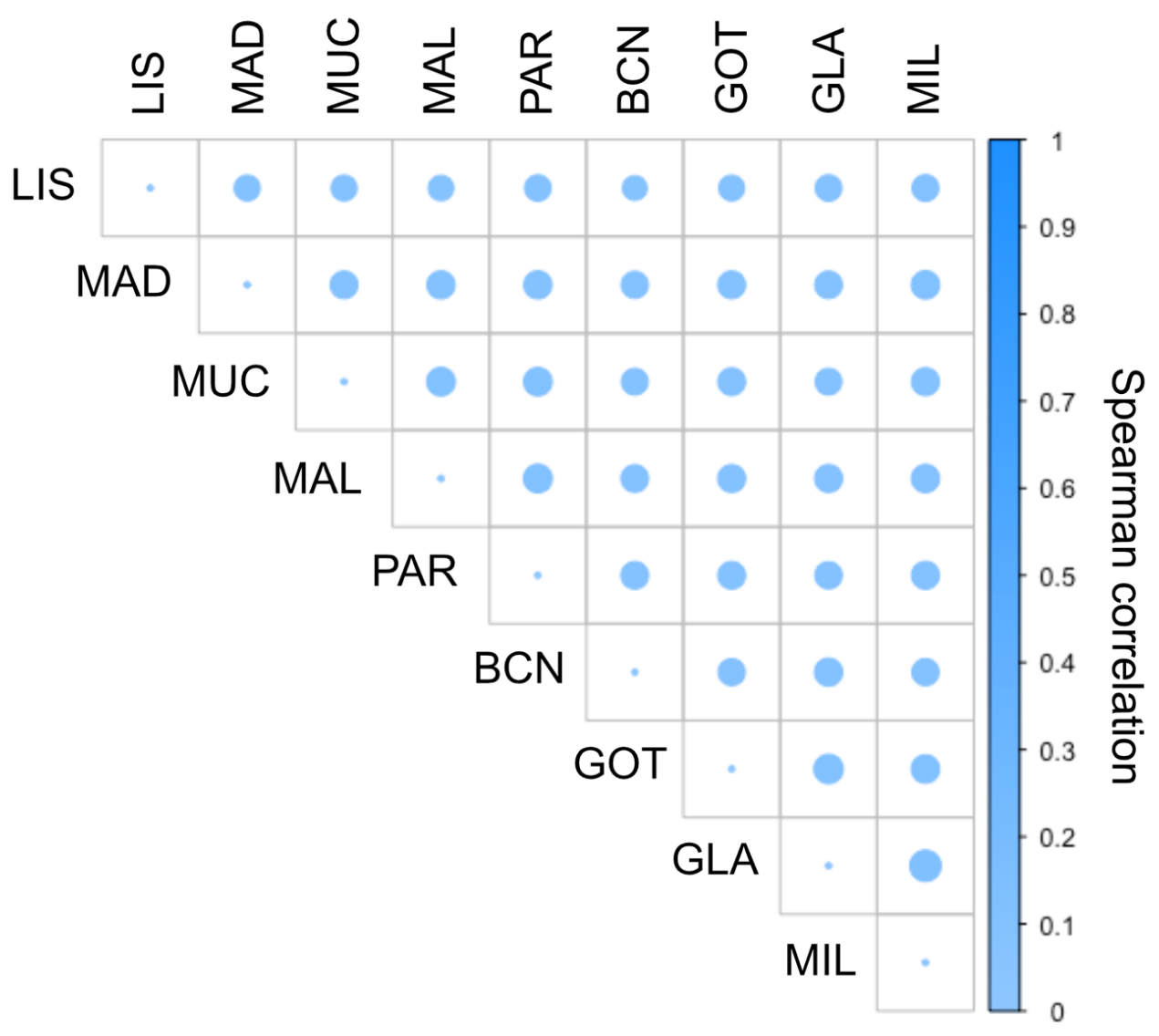

Fig. S10. Correlation plot of absolute Rsb values across populations. Shown are spearman correlations between absolute SNP-based $R s b$ values across all population pairs. Spearman correlations were uniformly low across populations, ranging from 0.08 to 0.013 . 

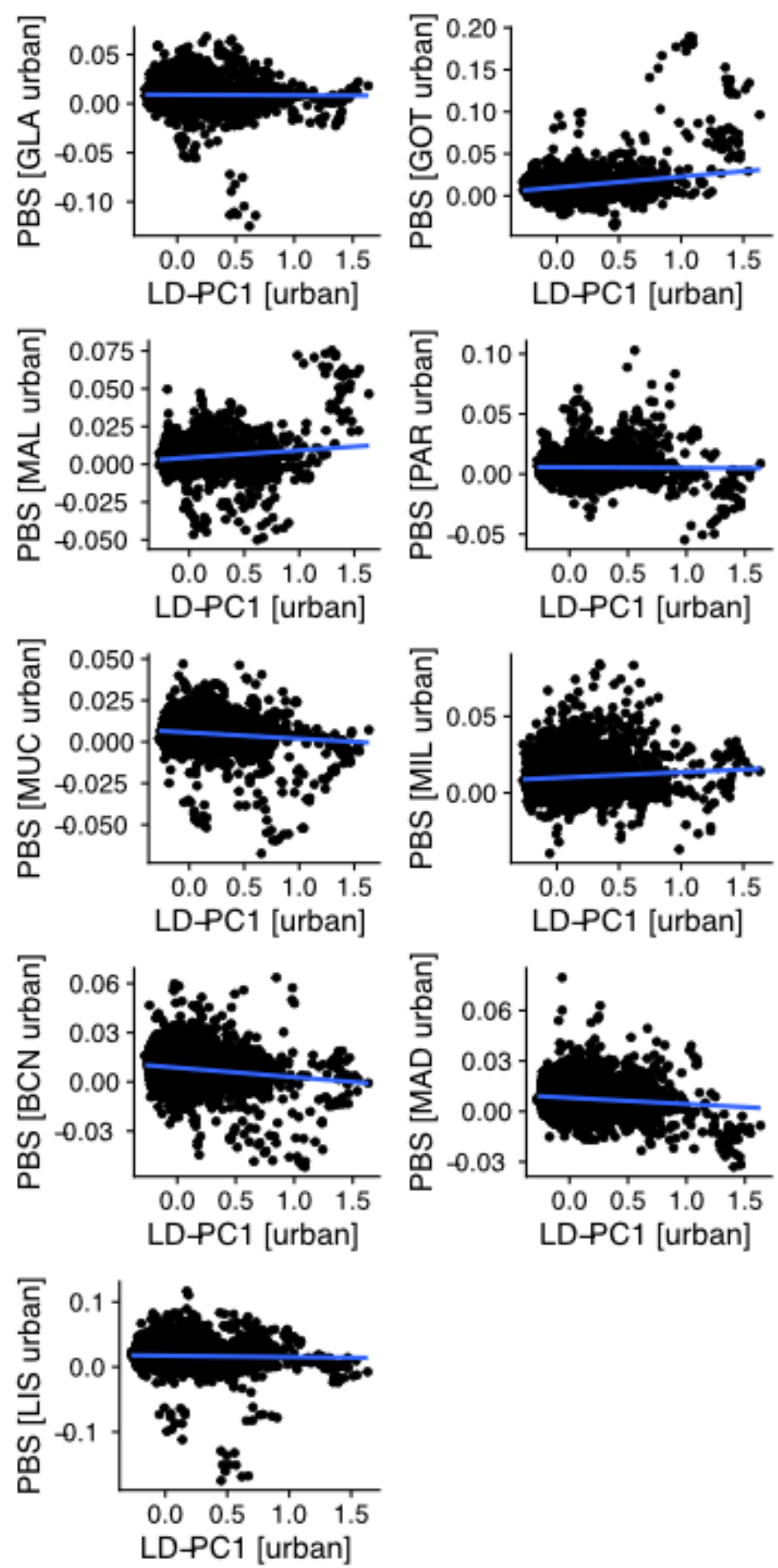

Fig. S11. Correlation of $P B S$ and $L D_{u r b}-P C 1$ for each population. LD was calculated and summarised across all urban populations. 

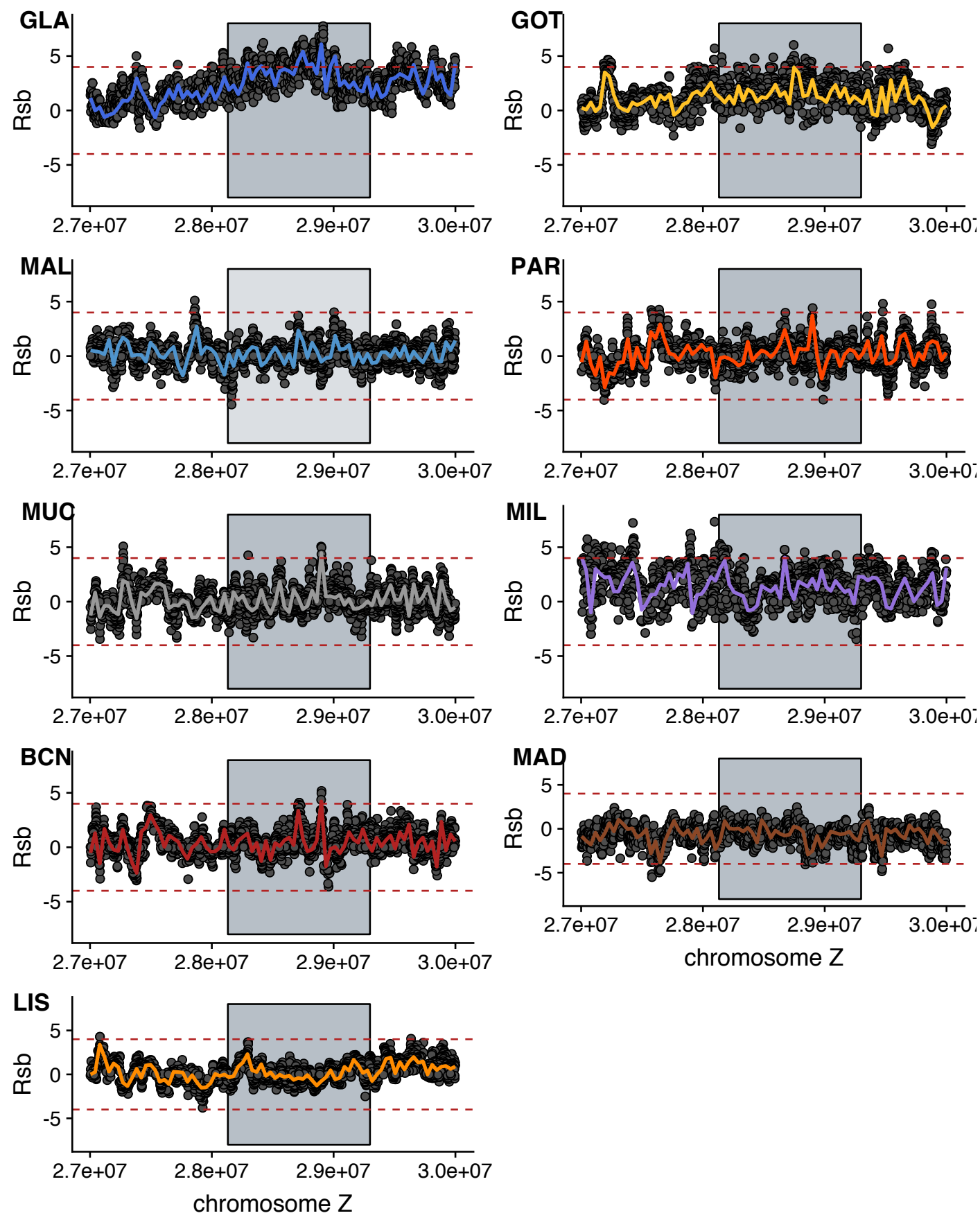

Fig. S12. Signatures of selection around the PTPRD gene. $R s b$ scores for each SNP around and within the PTPRD gene (grey box) on the $\mathrm{Z}$ chromosome by locality, including loess smoothed values (span $=$ 0.2 ). The upper and lower dashed lines show significance thresholds for signs of selection, respectively. BCN: Barcelona; GLA: Glasgow; GOT: Gothenburg; LIS: Lisbon; MAD: Madrid; MAL: Malmö; MIL: Milan; MUC: Munich; PAR: Paris. 


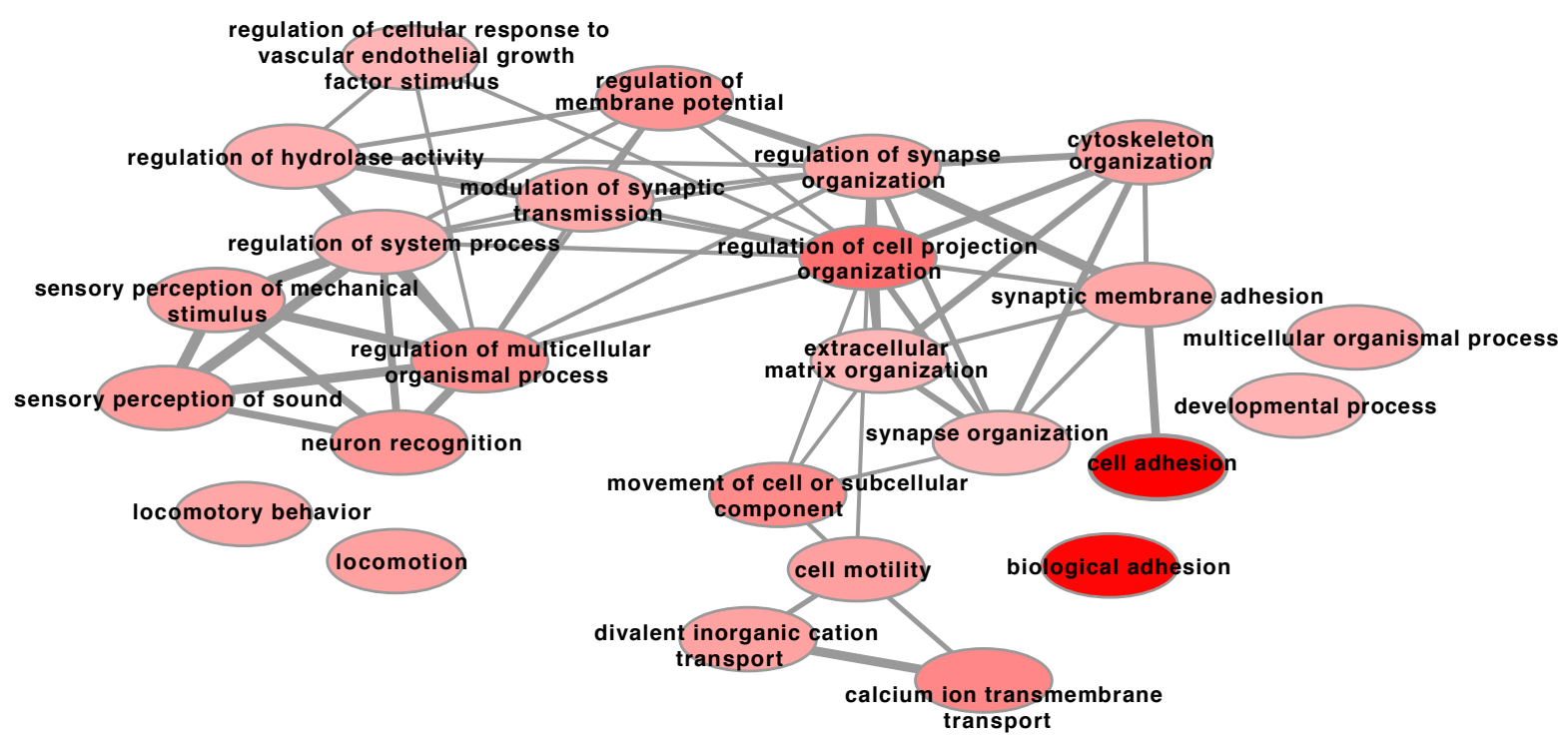

Fig. S13. Network of urbanisation associated (LFMM) gene ontology (GO) terms (biological processes). The network shows the relationship of GO terms associated with urban-associated genes based on the number of shared genes between GO terms. The number of shared genes is given by the thickness of the grey lines. The colour intensity shows the degree of enrichment based on the p-value, with the highest intensity showing the lowest p-value. $28 \mathrm{GO}$ terms were enriched at a false discovery rate (FDR) $<0.05$ and $36 \mathrm{GO}$ terms had an FDR $>0.05$ after correction for multiple testing with FDR values ranging from $1.87 \times 10^{-7}$ to $2.23 \times 10^{-1}$. 
Table S1. Locality (city name), year of sampling, season, centred geographical coordinates per site (urban/rural populations), urbanisation degree ( $\mathrm{PC}_{\mathrm{Urb}}$, positive values indicate higher urbanisation intensity), number of genotyped individuals $(\mathrm{n})$, expected heterozygosity $\left(\mathrm{H}_{\mathrm{e}}\right)$, pairwise genetic differentiation $\left(\mathrm{F}_{\mathrm{ST}}\right)$ and distance between the urban and rural populations for each of the studied localities. Cities are sorted in alphabetical order.

\begin{tabular}{|c|c|c|c|c|c|c|c|c|c|c|c|c|}
\hline \multirow[b]{2}{*}{ City } & \multirow[b]{2}{*}{ Year } & \multirow[b]{2}{*}{ Season } & \multicolumn{3}{|c|}{ Urban } & \multicolumn{4}{|c|}{ Rural } & \multirow[b]{2}{*}{$\mathbf{H}_{\mathbf{e}}$} & \multirow[b]{2}{*}{$F_{S T}$} & \multirow[b]{2}{*}{$\begin{array}{c}\text { Distance } \\
(\mathbf{k m})\end{array}$} \\
\hline & & & Coordinates & $\mathbf{P C}_{\text {Urb }}$ & $n$ & $\mathbf{H}_{\mathbf{e}}$ & Coordinates & $P C_{U r b}$ & $n$ & & & \\
\hline Barcelona & 2015 & Winter & $\begin{array}{c}41^{\circ} 23^{\prime} 24.0^{\prime \prime} \mathrm{N} \\
2^{\circ} 11^{\prime} 24.0 " \mathrm{E}\end{array}$ & 2.00 & 10 & 0.341 & $\begin{array}{c}41^{\circ} 42^{\prime} 00.0^{\prime \prime} \mathrm{N} \\
2^{\circ} 21^{\prime} 36.0^{\prime \prime} \mathrm{E}\end{array}$ & -2.29 & 10 & 0.348 & 0.012 & 5 \\
\hline Glasgow & 2015 & Breeding & $\begin{array}{c}55^{\circ} 52^{\prime} 48.0 " \mathrm{~N} \\
4^{\circ} 15^{\prime} 36.0^{\prime \prime} \mathrm{W}\end{array}$ & 2.96 & 10 & 0.337 & $\begin{array}{l}56^{\circ} 07^{\prime} 12.0^{\prime \prime} \mathrm{N} \\
4^{\circ} 35^{\prime} 24.0^{\prime \prime} \mathrm{W}\end{array}$ & -1.71 & 10 & 0.344 & 0.013 & 33 \\
\hline Gothenburg & 2015 & Breeding & $\begin{array}{l}57^{\circ} 41^{\prime} 24.0^{\prime \prime} \mathrm{N} \\
11^{\circ} 56^{\prime} 24.0^{\prime \prime} \mathrm{E}\end{array}$ & 2.13 & 10 & 0.342 & $\begin{array}{l}57^{\circ} 30 ' 00.0^{\prime \prime} \mathrm{N} \\
12^{\circ} 00^{\prime} 36.0^{\prime \prime} \mathrm{E}\end{array}$ & -2.39 & 10 & 0.350 & 0.016 & 25 \\
\hline Lisbon & 2014 & $\begin{array}{l}\text { Post- } \\
\text { Breeding }\end{array}$ & $\begin{array}{l}38^{\circ} 44^{\prime} 24.0^{\prime \prime} \mathrm{N} \\
9^{\circ} 10^{\prime} 48.0^{\prime \prime} \mathrm{W}\end{array}$ & 0.65 & 10 & 0.321 & $\begin{array}{l}38^{\circ} 51^{\prime} 36.0^{\prime \prime} \mathrm{N} \\
8^{\circ} 49^{\prime} 48.0^{\prime \prime} \mathrm{W}\end{array}$ & -1.86 & 10 & 0.333 & 0.050 & 33 \\
\hline Madrid & 2014 & Breeding & $\begin{array}{c}40^{\circ} 26^{\prime} 24.0^{\prime \prime} \mathrm{N} \\
3^{\circ} 43^{\prime} 48.0^{\prime \prime} \mathrm{W}\end{array}$ & 1.60 & 10 & 0.344 & $\begin{array}{c}40^{\circ} 34^{\prime} 12.0^{\prime \prime} \mathrm{N} \\
4^{\circ} 09^{\prime} 36.0^{\prime \prime} \mathrm{W}\end{array}$ & -2.05 & 10 & 0.348 & 0.013 & 39 \\
\hline Malmö & 2013 & Breeding & $\begin{array}{l}55^{\circ} 36^{\prime} 00.0^{\prime \prime} \mathrm{N} \\
12^{\circ} 59^{\prime} 24.0^{\prime \prime} \mathrm{E}\end{array}$ & 2.32 & 16 & 0.356 & $\begin{array}{l}55^{\circ} 39^{\prime} 00.0^{\prime \prime} \mathrm{N} \\
13^{\circ} 34^{\prime} 12.0^{\prime \prime} \mathrm{E}\end{array}$ & -2.44 & 16 & 0.355 & 0.009 & 37 \\
\hline Milan & 2014 & $\begin{array}{l}\text { Post- } \\
\text { Breeding }\end{array}$ & $\begin{array}{c}45^{\circ} 31^{\prime} 48.0 " \mathrm{~N} \\
9^{\circ} 12^{\prime} 36.0 " \mathrm{E}\end{array}$ & 1.49 & 10 & 0.342 & $\begin{array}{c}45^{\circ} 49^{\prime} 12.0^{\prime \prime} \mathrm{N} \\
9^{\circ} 17^{\prime} 24.0 " \mathrm{E}\end{array}$ & -2.24 & 10 & 0.343 & 0.026 & 34 \\
\hline Munich & 2015 & Winter & $\begin{array}{c}48^{\circ} 07^{\prime} 48.0^{\prime \prime} \mathrm{N} \\
1^{\circ} 34^{\prime} 12.0^{\prime \prime} \mathrm{E}\end{array}$ & 3.39 & 10 & 0.350 & $\begin{array}{l}47^{\circ} 58^{\prime} 12.0^{\prime \prime} \mathrm{N} \\
11^{\circ} 14^{\prime} 24.0^{\prime \prime} \mathrm{E}\end{array}$ & -1.36 & 10 & 0.351 & 0.004 & 30 \\
\hline Paris & 2014 & Breeding & $\begin{array}{c}48^{\circ} 52^{\prime} 12.0^{\prime \prime N} \\
2^{\circ} 10^{\prime} 48.0^{\prime \prime} \mathrm{E}\end{array}$ & 2.36 & 10 & 0.351 & $\begin{array}{c}48^{\circ} 18^{\prime} 00.0^{\prime \prime} \mathrm{N} \\
2^{\circ} 39^{\prime} 36.0^{\prime \prime} \mathrm{E}\end{array}$ & -2.58 & 10 & 0.351 & 0.004 & 70 \\
\hline
\end{tabular}

Notes: Expected heterozygosity is significantly lower in urban compared to rural great tits in Glasgow, Gothenburg Barcelona, Madrid and Lisbon based on t-tests ( $<$ 0.05). 
Table S2. Genes associated with "core urbanisation SNPs".

\section{Gene symbol Gene name/ Description}

\section{Selection}

\begin{tabular}{lll}
\hline FARP1 & FERM, ARH/RhoGEF and pleckstrin domain protein 1 & (N. localities) \\
CELF2 & CUGBP Elav-like family member 2 & 1 \\
IQGAP2 & IQ motif containing GTPase activating protein 2 & 0 \\
RNF38 & ring finger protein 38 & 1 \\
DACH1 & dachshund family transcription factor 1 & 2 \\
DHCR24 & 24-dehydrocholesterol reductase & 0 \\
SV2C & synaptic vesicle glycoprotein 2C & 0 \\
AVL9 & AVL9 cell migration associated & 1 \\
CADM2 & cell adhesion molecule 2 & 0 \\
GABRG3 & gamma-aminobutyric acid type A receptor gamma3 subunit & 0 \\
DNAl1 & dynein axonemal intermediate chain 1 & 0 \\
NTRK2 & neurotrophic receptor tyrosine kinase 2 & 2 \\
ABCB1 & ATP binding cassette subfamily B member 1 & 4 \\
ELP6 & elongator acetyltransferase complex subunit 6 & 0 \\
ADAMTS12 & ADAM metallopeptidase with thrombospondin type 1 motif 12 & 0 \\
\hline
\end{tabular}

Notes: Selection (N.localities) - Number of urban populations (localities) a particular gene is under selection in based on the haplotypebased selection analysis (Rsb value) 
Table S3. Genes putatively under selection in at least four cities based on $P B S$ and/or $R s b$. The number gives the number of cities in which a gene was detected to be under selection based on the given summary statistic.

\begin{tabular}{|c|c|c|c|}
\hline Gene symbol & Gene name/Description & PBS & Rsb \\
\hline PTPRD & Protein Tyrosine Phosphatase, Receptor Type D & 6 & 8 \\
\hline GNAQ & G protein subunit alpha $q$ & 6 & 5 \\
\hline FOCAD & Focadhesin & 5 & 8 \\
\hline $\mathrm{CDH} 18$ & Cadherin 18 & 5 & 5 \\
\hline HACD4 & 3-hydroxyacyl-CoA dehydratase 4 & 5 & 4 \\
\hline GLIS3 & GLIS family zinc finger 3 & 4 & 7 \\
\hline MLLT3 & MLLT3 super elongation complex subunit & 4 & 6 \\
\hline PIP5K1B & $\begin{array}{l}\text { Phosphatidylinositol-4-phosphate 5-kinase type } 1 \\
\text { beta }\end{array}$ & 4 & 6 \\
\hline PRUNE2 & Prune homolog 2 with $\mathrm{BCH}$ domain & 4 & 5 \\
\hline VPS13A & Vacuolar protein sorting 13 homolog $A$ & 4 & 5 \\
\hline$A R I D 3 C$ & AT-rich interaction domain $3 \mathrm{C}$ & 4 & 4 \\
\hline DCTN3 & Dynactin subunit 3 & 4 & 4 \\
\hline DNAl1 & Dynein axonemal intermediate chain 1 & 4 & 4 \\
\hline ENHO & Energy homeostasis associated & 4 & 4 \\
\hline FXN & Frataxin & 4 & 4 \\
\hline GALT & Galactose-1-phosphate uridylyltransferase & 4 & 4 \\
\hline LOC107215801 & Uncharacterized & 4 & 4 \\
\hline LOC107215987 & Uncharacterized & 4 & 4 \\
\hline$R P P 25 L$ & Ribonuclease P/MRP subunit p25 like & 4 & 4 \\
\hline SIGMAR1 & Sigma non-opioid intracellular receptor 1 & 4 & 4 \\
\hline TJP2 & Tight junction protein 2 & 4 & 4 \\
\hline CEP78 & Centrosomal protein 78 & 4 & 3 \\
\hline FOXB2 & Forkhead box B2 & 4 & 3 \\
\hline FREM1 & FRAS1 related extracellular matrix 1 & 4 & 3 \\
\hline ITGA2 & Integrin subunit alpha 2 & 4 & 3 \\
\hline LOC107198290 & Uncharacterized & 4 & 3 \\
\hline LOC107216175 & Uncharacterized & 4 & 3 \\
\hline LOC107216192 & Molybdopterin synthase sulfur carrier subunit-like & 4 & 3 \\
\hline LOC107216193 & Molybdopterin synthase catalytic subunit & 4 & 3 \\
\hline LOC107216195 & Uncharacterized & 4 & 3 \\
\hline LOC107216233 & Uncharacterized & 4 & 3 \\
\hline LOC107216234 & Uncharacterized & 4 & 3 \\
\hline LOC107216235 & Uncharacterized & 4 & 3 \\
\hline SNAPC3 & $\begin{array}{l}\text { Small nuclear RNA activating complex } \\
\text { polypeptide } 3\end{array}$ & 4 & 3 \\
\hline LOC107198252 & $\begin{array}{l}\text { Killer cell lectin-like receptor subfamily B member } \\
\text { 1B allele B }\end{array}$ & 3 & 7 \\
\hline
\end{tabular}




\begin{tabular}{llll} 
LOC107198253 & Butyrophilin subfamily 1 member A1-like & 3 & 7 \\
LOC107198254 & C-type lectin domain family 2 member B-like & 3 & 7 \\
LOC107198255 & E3 ubiquitin-protein ligase TRIM7-like & 3 & 7 \\
LOC107215749 & Uncharacterized & 3 & 7 \\
LOC107215825 & Glutamine-rich protein 2-like & 3 & 7 \\
PRR16 & Proline rich 16 & 3 & 7 \\
SLC1A1 & SLC1A1 & 3 & 7 \\
AP3B1 & AP3B1 & 3 & 6 \\
LOC107198413 & Uncharacterized & 3 & 5 \\
LOC107216267 & Uncharacterized & 3 & 5 \\
AKT3 & AKT serine/threonine kinase 3 & 3 & 4 \\
CCDC171 & Coiled-coil domain containing 171 & 3 & 4 \\
COL4A6 & COL4A6 & 3 & 4 \\
KDM4C & Lysine demethylase 4C & 3 & 4 \\
LOC107198356 & Uncharacterized & 3 & 4 \\
LOC107215808 & Interferon kappa-like & 3 & 4 \\
LOC107216174 & Phosphodiesterase 4D & 3 & 4 \\
NFIB & Nuclear factor I B & 3 & 4 \\
PSIP1 & PC4 and SFRS1 interacting protein 1 & 3 & 4 \\
\hline
\end{tabular}


Table S4. Overrepresented GO terms for genes associated with urbanisation in the LFMM and BayPass analysis.

\begin{tabular}{|c|c|c|c|c|c|c|}
\hline $\begin{array}{l}\text { GO } \\
\text { category }\end{array}$ & GO.ID & Description & Obs. & $\begin{array}{l}\text { Fold } \\
\text { enriched }\end{array}$ & p-value & FDR \\
\hline Processes & GO:0098742 & $\begin{array}{l}\text { cell-cell adhesion via plasma-membrane } \\
\text { adhesion molecules }\end{array}$ & 38 & 2.920 & $8.27 \mathrm{E}-10$ & $7.03 \mathrm{E}-07$ \\
\hline Processes & GO:0050808 & synapse organization & 55 & 2.078 & 1.04E-07 & 4.43E-05 \\
\hline Processes & GO:0050803 & regulation of synapse structure or activity & 35 & 2.312 & 1.86E-06 & 4.36E-04 \\
\hline Processes & GO:0016358 & dendrite development & 36 & 2.272 & 2.05E-06 & 4.36E-04 \\
\hline Processes & GO:0042391 & regulation of membrane potential & 52 & 1.907 & $3.50 \mathrm{E}-06$ & $5.95 \mathrm{E}-04$ \\
\hline Processes & GO:0050954 & sensory perception of mechanical stimulus & 24 & 2.399 & 4.06E-05 & $5.03 \mathrm{E}-03$ \\
\hline Processes & GO:0019932 & second messenger-mediated signalling & 46 & 1.823 & 4.14E-05 & $5.03 E-03$ \\
\hline Processes & GO:0031346 & positive regulation of cell projection organization & 46 & 1.811 & 4.94E-05 & 5.25E-03 \\
\hline Processes & GO:0010975 & regulation of neuron projection development & 56 & 1.691 & $5.63 \mathrm{E}-05$ & $5.32 \mathrm{E}-03$ \\
\hline Processes & GO:0022604 & regulation of cell morphogenesis & 52 & 1.650 & 1.96E-04 & 1.63E-02 \\
\hline Functions & GO:0045503 & dynein light chain binding & 8 & 4.440 & $1.98 \mathrm{E}-04$ & $3.08 \mathrm{E}-02$ \\
\hline Functions & GO:0030507 & spectrin binding & 8 & 4.230 & 2.95E-04 & $3.08 \mathrm{E}-02$ \\
\hline Functions & GO:0050839 & cell adhesion molecule binding & 52 & 1.610 & 3.34E-04 & $3.08 \mathrm{E}-02$ \\
\hline Functions & GO:0005201 & extracellular matrix structural constituent & 23 & 2.090 & 4.86E-04 & 3.36E-02 \\
\hline Functions & GO:0019199 & transmembrane receptor protein kinase activity & 15 & 2.520 & $6.22 \mathrm{E}-04$ & $3.44 \mathrm{E}-02$ \\
\hline Functions & GO:0019838 & growth factor binding & 19 & 2.200 & 7.95E-04 & 3.67E-02 \\
\hline Functions & GO:0005518 & collagen binding & 12 & 2.560 & $1.85 \mathrm{E}-03$ & 7.33E-02 \\
\hline Functions & GO:0051959 & dynein light intermediate chain binding & 7 & 3.530 & $2.40 \mathrm{E}-03$ & 8.07E-02 \\
\hline Functions & GO:0008066 & glutamate receptor activity & 7 & 3.380 & $3.18 \mathrm{E}-03$ & 8.07E-02 \\
\hline Functions & GO:0045505 & dynein intermediate chain binding & 7 & 3.380 & $3.18 \mathrm{E}-03$ & 8.07E-02 \\
\hline Component & GO:0097060 & synaptic membrane & 67 & 2.272 & $5.21 \mathrm{E}-11$ & 8.86E-09 \\
\hline Component & GO:0098984 & neuron to neuron synapse & 52 & 2.208 & $2.45 \mathrm{E}-08$ & $2.08 \mathrm{E}-06$ \\
\hline Component & GO:0099572 & postsynaptic specialization & 48 & 2.061 & 7.54E-07 & 4.27E-05 \\
\hline Component & GO:0098978 & glutamatergic synapse & 48 & 1.924 & $5.68 \mathrm{E}-06$ & $2.00 \mathrm{E}-04$ \\
\hline Component & GO:0044309 & neuron spine & 28 & 2.450 & $5.89 \mathrm{E}-06$ & $2.00 \mathrm{E}-04$ \\
\hline Component & GO:0033267 & axon part & 47 & 1.839 & 2.43E-05 & $6.89 \mathrm{E}-04$ \\
\hline Component & GO:0042383 & sarcolemma & 22 & 2.473 & 5.06E-05 & $1.23 E-03$ \\
\hline Component & GO:0098793 & presynapse & 53 & 1.692 & 7.69E-05 & $1.64 \mathrm{E}-03$ \\
\hline Component & GO:0031594 & neuromuscular junction & 15 & 2.915 & 1.14E-04 & $2.16 \mathrm{E}-03$ \\
\hline Component & GO:0043235 & receptor complex & 43 & 1.712 & 2.87E-04 & 4.88E-03 \\
\hline
\end{tabular}

Notes: Obs. (Total) - observed number of genes associated with gene ontology term. 